\title{
Sterically Directed Functionalization of Aromatic C-H Bonds: Selective Borylation ortho to Cyano Groups in Arenes and Heterocycles.
}

\author{
Ghayoor A. Chotana, Michael A. Rak, and Milton R. Smith, III* \\ Department of Chemistry, Michigan State University, East Lansing, MI 48824 USA \\ smithmil@msu.edu
}

\section{Supporting Information}

\section{Methods:}

Pinacolborane (HBPin) and bis $\left(\eta^{4}-1,5\right.$-cyclooctadiene)-di- $\mu$-methoxy-diiridium(I) $\{\operatorname{Ir}(\mathrm{OMe})(\mathrm{COD})\}_{2}$ were prepared per literature procedures. ${ }^{1,2}$ Bis(pinacolato)diboron $\left(\mathrm{B}_{2} \mathrm{Pin}_{2}\right)$ was purchased from Callery Chemical Company and was used without purification. Tris(dibenzylidineacetone)dipalladium $(0)\left(\mathrm{Pd}_{2} \mathrm{dba}_{3}\right)$ was purchased from Strem. 4,4'-Di-t-butyl-2,2'-bipyridine ( $\mathrm{d}^{\prime}$ bpy), tricyclohexylphosphine, and potassium acetate were purchased from Aldrich. 1-Bromo-3,5-difluorobenzene, 2-bromo-1,3-difluorobenzene, 1-bromo-2,4-difluorobenzene, and 4-iodobenzonitrile were purchased from Alfa Aesar. 4-Bromobenzonitrile and 4-methoxybenzonitrile were purchased from Lancaster Synthesis. 2-Bromo-4-methylbenzonitrile was purchased from Trans World Chemicals. 5-Bromo-2-cyanopyridine and 2-bromo-5-cyanopyridine were purchased from Matrix Scientific. All other benzonitriles were purchased from Aldrich. All substrates were purified before use. Solid substrates were sublimed under vacuum. 5-Methyl-2-furonitrile and 5-bromothiophene-2-carbonitrile were passed through activated alumina. 1,4-Dioxane and $n$-hexane were refluxed over sodium, distilled, and degassed. Tetrahydrofuran was used from a dry still packed with activated alumina. Silica gel was purchased from EM Science (230-400 Mesh).

Unless otherwise specified, all reactions were carried out at $25{ }^{\circ} \mathrm{C}$ in $20 \mathrm{~mL}$ vials in a glove box under a nitrogen atmosphere. All reactions were monitored by a Varian CP-3800 GC-FID (column type: WCOT Fused silica $30 \mathrm{~m} \times 0.25 \mathrm{~mm}$ ID coating CP-SIL 8 CB). GC-FID method: $70{ }^{\circ} \mathrm{C}, 2 \mathrm{~min} . ; 20{ }^{\circ} \mathrm{C} / \mathrm{min}, 9 \mathrm{~min}$.; $250{ }^{\circ} \mathrm{C}, 20 \mathrm{~min} . ; 1.8 \mathrm{~mL} / \mathrm{min}$ flow rate. All reported yields are for isolated materials. 
${ }^{1} \mathrm{H}$ and ${ }^{13} \mathrm{C}$ NMR spectra were recorded on a Varian Inova-300 (300.11 and 75.47 MHz respectively), Varian VXR-500, or Varian Unity-500-plus spectrometer (499.74 and 125.67 MHz respectively) and referenced to residual solvent signals. ${ }^{11} \mathrm{~B}$ spectra were recorded on a Varian VXR-300 operating at $96.29 \mathrm{MHz}$ and were referenced to neat $\mathrm{BF}_{3} \cdot \mathrm{Et}_{2} \mathrm{O}$ as the external standard. ${ }^{19} \mathrm{~F}$ spectra were recorded on a Varian Inova-300 operating at $282.36 \mathrm{MHz}$ and were referenced to neat $\mathrm{CFCl}_{3}$ as the external standard. All coupling constants are apparent $J$ values measured at the indicated field strengths. All 1-dimensional NOE experiments were obtained using the Varian implementation of the DPFGSE-NOE experiment ${ }^{3}$ (hereafter termed NOESY1D). All 2-dimensional experiments were run using $z$-axis pulse field gradients. Elemental analyses were performed at Michigan State University using a Perkin Elmer Series II 2400 CHNS/O Analyzer. GC-MS data were obtained using a Varian Saturn 2200 GC/MS (column type: WCOT Fused silica $30 \mathrm{~m} \times 0.25 \mathrm{~mm}$ ID coating CP-SIL $8 \mathrm{CB}$ ). High-resolution mass spectra were obtained at the Michigan State University Mass Spectrometry Service Center with a JOEL-AX505 mass spectrometer (resolution 7000). Melting points were measured on a MEL-TEMP ${ }^{\circledR}$ capillary melting apparatus and are uncorrected.

\section{General Procedure}

Substrates which gave a significant amount of both isomers were borylated employing an excess of benzonitrile to minimize diborylation (General procedure A), otherwise, excess borane was employed (General procedure B). Unless otherwise specified, all reactions were run in THF solutions at $25{ }^{\circ} \mathrm{C}$ with $3 \mathrm{~mol} \%$ [Ir]. The major isomer for the borylation of 4-methylbenzonitrile was identified by preparing an authentic sample using a slightly modified literature procedure. ${ }^{4}$ The regioisomers in all other cases were assigned by NMR spectroscopy $\left({ }^{13} \mathrm{C}\right.$ for substrates which have fluorine, while gHMBC and NOESY1D were used for substrates without fluorine). Ratios of the major versus minor isomer were determined in the crude reaction mixtures. Yields are based on the limiting reagent. 


\section{General Procedure A (Borane as limiting reactant):}

In a glove box, a $20 \mathrm{~mL}$ vial, equipped with a magnetic stirring bar, was charged with $\{\mathrm{Ir}(\mathrm{OMe})(\mathrm{COD})\}_{2}(5.0 \mathrm{mg}, 0.0075 \mathrm{mmol}, 3 \mathrm{~mol} \% \mathrm{Ir}), 4,4^{\prime}$-di-t-butyl-2,2'-bipyridine (d $\mathrm{d}^{\mathrm{b}} \mathrm{by}$ ) (4.0 mg, $0.015 \mathrm{mmol}, 3 \mathrm{~mol} \%$ ), and pinacolborane (HBPin) (73 $\mu \mathrm{L}, 64 \mathrm{mg}, 0.5$ mmol, 1 equivalent). These reagents were dissolved in $2 \mathrm{~mL}$ of THF, the corresponding benzonitrile ( 2 mmol, 4 equivalents) was added, and the mixture was stirred at room temperature until the reaction was judged complete by GC-FID. Solvent was removed under reduced pressure. The crude material was dissolved in $\mathrm{CH}_{2} \mathrm{Cl}_{2}$ and passed through a plug of silica gel to remove metal byproducts. Kugelrohr distillation gave analytically pure samples.

\section{General Procedure B (Benzonitrile as limiting reactant):}

In a glove box, a $20 \mathrm{~mL}$ vial, equipped with a magnetic stirring bar, was charged with $\{\mathrm{Ir}(\mathrm{OMe})(\mathrm{COD})\}_{2}(10 \mathrm{mg}, 0.015 \mathrm{mmol}, 3 \mathrm{~mol} \% \mathrm{Ir}), \mathrm{d}^{t}$ bpy $(8.1 \mathrm{mg}, 0.03 \mathrm{mmol}, 3$ mol\%), and excess HBPin or $\mathrm{B}_{2} \mathrm{Pin}_{2}$ (1.1 to 3.2 equivalents of boron). These reagents were dissolved in $3 \mathrm{~mL}$ of THF, the corresponding benzonitrile ( $1 \mathrm{mmol}, 1$ equivalent) was added, and the mixture was stirred at room temperature until the reaction was judged complete by GC-FID. Solvent was removed under reduced pressure. The crude material was dissolved in $\mathrm{CH}_{2} \mathrm{Cl}_{2}$ and passed through a plug of silica gel to furnish the desired borylated product.

\section{General Procedure C (Diborylation):}

In a glove box, a $20 \mathrm{~mL}$ vial, equipped with a magnetic stirring bar, was charged with $\{\mathrm{Ir}(\mathrm{OMe})(\mathrm{COD})\}_{2}(20 \mathrm{mg}, 0.03 \mathrm{mmol}, 6 \mathrm{~mol} \% \mathrm{Ir}), \mathrm{d}^{t}$ bpy $(16.1 .1 \mathrm{mg}, 0.06 \mathrm{mmol}, 6$ mol\%), and excess HBPin (4 equivalent of boron). These reagents were dissolved in 3 $\mathrm{mL}$ of THF, the corresponding benzonitrile ( $1 \mathrm{mmol}, 1$ equivalent) was added, and the mixture was stirred at room temperature until the reaction was judged complete by GC-FID. Solvent was removed under reduced pressure. The crude material was dissolved in $\mathrm{CH}_{2} \mathrm{Cl}_{2}$ and passed through a plug of silica gel to furnish the desired borylated product. 


\section{Regioisomer assignment by NMR spectroscopy:}

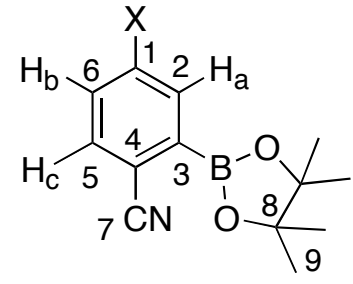

Isomer A

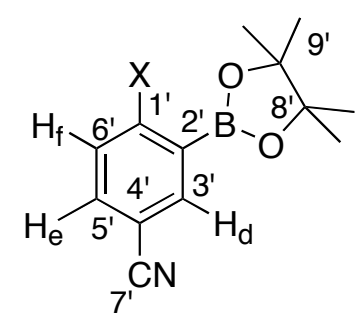

Isomer B

From gHMBC NMR experiments, the two regioisomers for the borylation of 4-substituted benzonitriles can be distinguished unambiguously. In Isomer A, carbon atoms represented as $\mathrm{C} 1$ and $\mathrm{C} 4$ on the benzene ring, as well as $\mathrm{C} 7$ (nitrile carbon) are the three quaternary carbon atoms in the $100-170$ ppm region (quaternary carbon $\mathrm{C} 3$ is typically not observed due to broadening from and coupling with boron). These three quaternary carbon atoms should show cross peaks due to long range $\mathrm{H}-\mathrm{C}$ couplings $\left({ }^{3} J_{\mathrm{C}-\mathrm{H}}\right)$, which can be observed using gHMBC spectroscopy. In the gHMBC spectrum, carbon atoms $\mathrm{C} 1$ and $\mathrm{C} 7$ should show one cross peak each to proton $\mathrm{H}_{\mathrm{c}}$, whereas carbon atom $\mathrm{C} 4$ should show two cross peaks to protons $\mathrm{H}_{\mathrm{a}}$ and $\mathrm{H}_{\mathrm{b}}$. Therefore the resulting number of cross peaks for $\mathrm{C} 1, \mathrm{C} 4$, and $\mathrm{C} 7$ should be 1,2 , and 1, respectively.

In Isomer B, carbon atoms represented as $\mathrm{C1}^{\prime}, \mathrm{C}^{\prime}$, on the benzene ring, as well as C7' (nitrile carbon) are the three quaternary carbon atoms in the 100-170 ppm region (quaternary carbon $\mathrm{C}^{\prime}$ is typically not observed due to broadening from and coupling with boron). These three quaternary carbon atoms should show cross peaks due to long range $\mathrm{H}-\mathrm{C}$ couplings $\left({ }^{3} J_{\mathrm{C}-\mathrm{H}}\right)$. In the gHMBC spectrum, carbon atoms $\mathrm{C}^{\prime}$ and $\mathrm{C} 7{ }^{\prime}$ should show two cross peaks each, to protons $\mathrm{H}_{\mathrm{d}}$ and $\mathrm{H}_{\mathrm{e}}$, whereas carbon atom $\mathrm{C}^{4}$ ' should show only one cross peak to proton $\mathrm{H}_{\mathrm{f}}$. Therefore the resulting number of cross peaks for $\mathrm{Cl}^{\prime}$, C4', and C7' should be 2, 1, and 2, respectively. Hence isomers A and B can be unambiguously assigned from gHMBC data.

For isomer $\mathrm{A}$, with proton $\mathrm{H}_{\mathrm{c}}$ unambiguously assigned by $\mathrm{gHMBC}, \mathrm{H}_{\mathrm{a}}$ and $\mathrm{H}_{\mathrm{b}}$ can be assigned from their multiplicities. Proton $\mathrm{H}_{\mathrm{a}}$ appears as a doublet, coupled to proton $\mathrm{H}_{\mathrm{b}}$ with $\mathrm{J} \approx 2-3 \mathrm{~Hz}$. Proton $\mathrm{H}_{\mathrm{b}}$ appears as a doublet of doublets due to coupling to protons $\mathrm{H}_{\mathrm{a}}$ and $\mathrm{H}_{\mathrm{c}}$. Carbon atoms $\mathrm{C} 2, \mathrm{C} 6$, and $\mathrm{C} 5$ were then assigned from the correlations in the gHMQC spectra. Carbon atom C7 (nitrile carbon) usually appears around $\delta 119$. 
Depending on the substituent, carbon atom $\mathrm{C} 1$ was usually found shifted downfield around $\delta 130-170$ (except in 4-iodobenzonitrile for which it appears around $\delta 100$ ). Carbon atom C4 is shifted upfield, and was usually found around $\delta 100-115$. Similarly, all the carbons of isomer $\mathrm{B}$ can be assigned.

In the five membered heterocycles, the ${ }^{4} J_{\mathrm{H}-\mathrm{H}}$ coupling was used together with gHMBC and NOESY1D spectroscopy to identify the major isomer. Regioisomers in the fluorine containing benzonitriles were assigned by ${ }^{13} \mathrm{C}$ spectroscopy (with the help of the fact that the boron bearing carbon is not observed due to broadening from and coupling with boron). In case of 1,3-dicyanobenzene, ${ }^{1} \mathrm{H}$ NMR spectroscopy was employed to assign the major and minor isomers.

\section{Experimental Details and spectroscopic data:}

Table 1, Entry 1: Borylation of 4-fluorobenzonitrile.

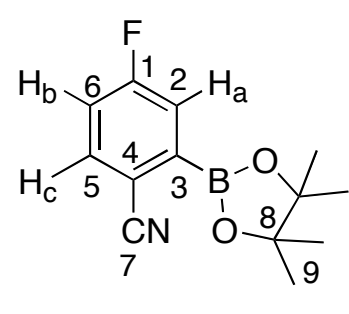

$11 \%$

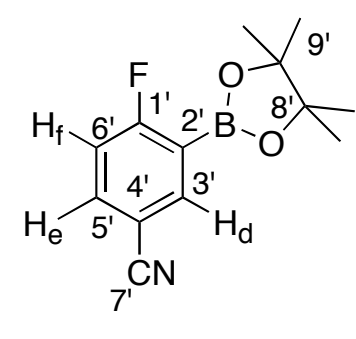

$89 \%$

General procedure A was applied to 4-fluorobenzonitrile (242 $\mathrm{mg}, 2 \mathrm{mmol}$ ) and HBPin $(73 \mu \mathrm{L}, 64 \mathrm{mg}, 0.5 \mathrm{mmol})$ with a reaction time of $8 \mathrm{~h}$. The ratio of the two isomers in the crude reaction mixture by GC was 11:89. Kugelrohr distillation furnished a mixture of the two isomeric borylated products $(88.5 \mathrm{mg}, 72 \%)$ as a white solid. The ratio of the two isomers in the isolated product by GC was 8:92. ${ }^{13} \mathrm{C}$ NMR spectroscopy was used to assign the major isomer as 4-fluoro-3-(4,4,5,5-tetramethyl-1,3,2-dioxaboryl)benzonitrile. ${ }^{1} \mathrm{H}$ NMR $\left(\mathrm{CDCl}_{3}, 500 \mathrm{MHz}\right): \delta$ (major isomer) $8.04\left(\mathrm{dd},{ }^{4} J_{\mathrm{H}-\mathrm{F}}=5.4 \mathrm{~Hz}, J=2.2 \mathrm{~Hz}, 1 \mathrm{H}\right.$, $\left.\mathrm{H}_{\mathrm{d}}\right), 7.7\left(\mathrm{ddd}, J=8.5,2.2 \mathrm{~Hz},{ }^{4} J_{\mathrm{H}-\mathrm{F}}=4.9 \mathrm{~Hz}, 1 \mathrm{H}, \mathrm{H}_{\mathrm{e}}\right), 7.1\left(\mathrm{t}, J=8.5 \mathrm{~Hz}, 1 \mathrm{H}, \mathrm{H}_{\mathrm{f}}\right), 1.32$ (br $\mathrm{s}, 12 \mathrm{H}),\left(\right.$ minor isomer) $7.67\left(\mathrm{dd}, J=8.8 \mathrm{~Hz},{ }^{4} J_{\mathrm{H}-\mathrm{F}}=4.9 \mathrm{~Hz}, 1 \mathrm{H}, \mathrm{H}_{\mathrm{c}}\right), 7.52\left(\mathrm{dd},{ }^{3} J_{\mathrm{H}-\mathrm{F}}=8.5\right.$ $\left.\mathrm{Hz}, J=2.9 \mathrm{~Hz}, 1 \mathrm{H}, \mathrm{H}_{\mathrm{a}}\right), 7.17\left(\mathrm{dt}, J=8.3,2.9 \mathrm{~Hz}, 1 \mathrm{H}, \mathrm{H}_{\mathrm{b}}\right) 1.34($ br s, $12 \mathrm{H}) ;{ }^{13} \mathrm{C} \mathrm{NMR}$ $\left\{{ }^{1} \mathrm{H}\right\}\left(\mathrm{CDCl}_{3}, 125 \mathrm{MHz}\right): \delta$ (major isomer) $169.0\left(\mathrm{~d},{ }^{1} J_{\mathrm{C}-\mathrm{F}}=261.3 \mathrm{~Hz}, \mathrm{C} 1^{\prime}\right), 141.6(\mathrm{~d}$, 
$\left.{ }^{3} J_{\mathrm{C}-\mathrm{F}}=9.6 \mathrm{~Hz}, \mathrm{C} 3^{\prime}\right), 137.0\left(\mathrm{~d},{ }^{3} J_{\mathrm{C}-\mathrm{F}}=10.5 \mathrm{~Hz}, \mathrm{C} 5^{\prime}\right), 117.9$ (nitrile C7 $\left.{ }^{\prime}\right), 116.7\left(\mathrm{~d},{ }^{2} J_{\mathrm{C}-\mathrm{F}}=\right.$ $\left.25.6 \mathrm{~Hz}, \mathrm{C} 6^{\prime}\right), 108.2\left(\mathrm{~d},{ }^{4} J_{\mathrm{C}-\mathrm{F}}=3.8 \mathrm{~Hz}, \mathrm{C} 4^{\prime}\right), 84.5\left(\mathrm{C} 8^{\prime}\right), 24.7\left(\mathrm{C}^{\prime}\right)$ ), (minor isomer) $164.2\left(\mathrm{~d},{ }^{1} J_{\mathrm{C}-\mathrm{F}}=257.1 \mathrm{~Hz}, \mathrm{C} 1\right), 135.9\left(\mathrm{~d},{ }^{3} J_{\mathrm{C}-\mathrm{F}}=8.8 \mathrm{~Hz}, \mathrm{C} 5\right), 122.8\left(\mathrm{~d},{ }^{2} J_{\mathrm{C}-\mathrm{F}}=21.0 \mathrm{~Hz}\right.$, C2), 118.5 (d, $\left.{ }^{2} J_{\mathrm{C}-\mathrm{F}}=22.2 \mathrm{~Hz}, \mathrm{C} 6\right), 118.1$ (nitrile C7), 113.1 (C4), 85.1 (C8), 24.7 (C9);

${ }^{11} \mathrm{~B}$ NMR $\left(\mathrm{CDCl}_{3}, 96 \mathrm{MHz}\right): \delta 29.92 ;{ }^{19} \mathrm{~F} \mathrm{NMR}\left(\mathrm{CDCl}_{3}, 282 \mathrm{MHz}\right): \delta$ (major isomer) -92.62 (m), (minor isomer) -104.84 (m); FT-IR (neat): 3076, 2982, 2934, 2231, 1608, 1487, 1429, 1412, 1373, 1350, 1236, 1143, 1070, 964, 852, 835, $571 \mathrm{~cm}^{-1}$; LRMS (\% rel. int.): $\mathrm{m} / \mathrm{e}$ (major isomer) $247 \mathrm{M}^{+}$(26), 232 (100), 205 (12), 188 (20), (minor isomer) 247 $\mathrm{M}^{+}$(29), 232 (97), 206 (100), 189 (74), 148 (97), 121 (25) ; Anal. Cacld for $\mathrm{C}_{13} \mathrm{H}_{15} \mathrm{BFNO}_{2}$ : C, 63.20; H, 6.12; N, 5.67. Found: $\mathrm{C}, 63.52 ; \mathrm{H}, 6.20 ; \mathrm{N}, 5.56$. HRMS Calcd for $\mathrm{C}_{13} \mathrm{H}_{15} \mathrm{BFNO}_{2}: 247.1180$. Found: 247.1171.

\section{Table 1, Entry 2: Borylation of 4-chlorobenzonitrile.}
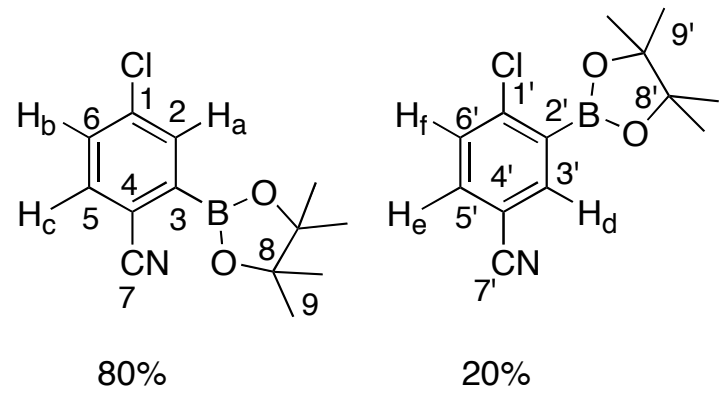

General procedure A was applied to 4-chlorobenzonitrile (550.3 mg, $4 \mathrm{mmol}$ ) and HBPin $(145 \mu \mathrm{L}, 128 \mathrm{mg}, 1 \mathrm{mmol})$ with a reaction time of $36 \mathrm{~h}$. The ratio of the two isomers in the crude reaction mixture by $\mathrm{GC}$ was 80:20. Kugelrohr distillation furnished a mixture of the two isomeric borylated products ( $200 \mathrm{mg}, 76 \%$ ) as a white solid. The ratio of the two isomers in the isolated product by GC was 81:19. gHMBC spectroscopy (Figure 1) was used to assign the major isomer as 4-chloro-2-(4,4,5,5-tetramethyl-1,3,2dioxaboryl)benzonitrile. ${ }^{1} \mathrm{H}$ NMR $\left(\mathrm{CDCl}_{3}, 300 \mathrm{MHz}\right.$ ): $\delta$ (major isomer) $7.80(\mathrm{~d}, J=2.2$ $\left.\mathrm{Hz}, 1 \mathrm{H}, \mathrm{H}_{\mathrm{a}}\right), 7.57$ (d, $J=8.3 \mathrm{~Hz}, 1 \mathrm{H}, \mathrm{H}_{\mathrm{c}}$ ), 7.45 (dd, $J=8.3,2.2 \mathrm{~Hz}, 1 \mathrm{H}, \mathrm{H}_{\mathrm{b}}$ ), 1.33 (br s, $12 \mathrm{H})$, (minor isomer) $7.94\left(\mathrm{~d}, J=2.2 \mathrm{~Hz}, 1 \mathrm{H}, \mathrm{H}_{\mathrm{d}}\right), 7.56\left(\mathrm{dd}, J=8.3,2.2 \mathrm{~Hz}, 1 \mathrm{H}, \mathrm{H}_{\mathrm{e}}\right.$ ), $7.41\left(\mathrm{~d}, J=8.3 \mathrm{~Hz}, 1 \mathrm{H}, \mathrm{H}_{\mathrm{f}}\right), 1.32(\mathrm{br} \mathrm{s}, 12 \mathrm{H}) ;{ }^{13} \mathrm{C} \mathrm{NMR}\left\{{ }^{1} \mathrm{H}\right\}\left(\mathrm{CDCl}_{3}, 75 \mathrm{MHz}\right): \delta$ (major isomer) 138.5 (C1), 135.8 (C2), 134.5 (C5), 131.2 (C6), 118.0 (nitrile C7), 115.3 (C4), 
85.0 (C8), 24.6 (C9), (minor isomer) $144.5\left(\mathrm{Cl}^{\prime}\right), 140.1$ (C3' ), 134.6 (C5' ), 130.2 (C6' ), 117.8 (nitrile $\mathrm{C}^{\prime}$ ), $110.2\left(\mathrm{C}^{\prime}\right), 84.7\left(\mathrm{C} 8^{\prime}\right), 24.6\left(\mathrm{C}^{\prime}\right) ;{ }^{11} \mathrm{~B} \mathrm{NMR}\left(\mathrm{CDCl}_{3}, 96 \mathrm{MHz}\right): \delta$ 29.59; FT-IR (neat): 2982, 2228, 1587, 1554, 1479, 1402, 1373, 1333, 1271, 1215, 1169, 1144, 1103, 1065, 1042, 965, 870, 847, $831 \mathrm{~cm}^{-1}$; LRMS (\% rel. int.): $\mathrm{m} / \mathrm{e}$ (major isomer) $263 \mathrm{M}^{+}$(24), 248 (65), 222 (100), 205 (31), 164(32), 137 (11), (minor isomer) $263 \mathrm{M}^{+}$ (1), 248 (27), 228 (100), 186 (60), 164 (15), 142 (6); Anal. Calcd for $\mathrm{C}_{13} \mathrm{H}_{15} \mathrm{BClNO}_{2}$ : C, 59.25; H, 5.74; N, 5.32. Found: C, 58.90; H, 5.74; N, 5.10.

\section{Table 1, Entry 3: Borylation of 4-Bromobenzonitrile.}
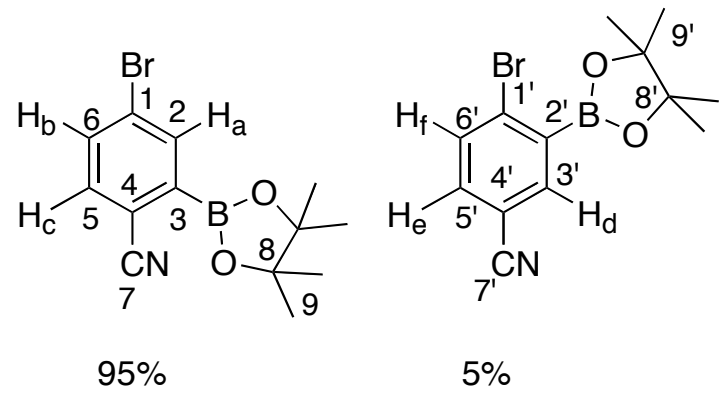

General procedure A was applied to 4-bromobenzonitrile (364.1 $\mathrm{mg}, 2 \mathrm{mmol}$ ) and HBPin ( $73 \mu \mathrm{L}, 64 \mathrm{mg}, 0.5 \mathrm{mmol})$ with a reaction time of $48 \mathrm{~h}$. The ratio of the two isomers in the crude reaction mixture by GC was 95:5. Kugelrohr distillation furnished a mixture of the two isomeric borylated products $(112 \mathrm{mg}, 73 \%)$ as a white solid. The ratio of the two isomers in the isolated product by GC was 97:3. gHMBC spectroscopy (Figure 2) was used to assign the major isomer as 4-bromo-2-(4,4,5,5-tetramethyl1,3,2-dioxaboryl)benzonitrile. ${ }^{1} \mathrm{H}$ NMR $\left(\mathrm{CDCl}_{3}, 500 \mathrm{MHz}\right.$ ): $\delta$ (major isomer) 7.97 (d, $J=$ $2.0 \mathrm{~Hz}, 1 \mathrm{H}, \mathrm{H}_{\mathrm{a}}$ ), $7.62\left(\mathrm{dd}, J=8.3,2.0 \mathrm{~Hz}, 1 \mathrm{H}, \mathrm{H}_{\mathrm{b}}\right), 7.50$ (d, $\left.J=8.3 \mathrm{~Hz}, 1 \mathrm{H}, \mathrm{H}_{\mathrm{c}}\right), 1.33$ (br s, $12 \mathrm{H}) ;{ }^{13} \mathrm{C}$ NMR $\left\{{ }^{1} \mathrm{H}\right\}\left(\mathrm{CDCl}_{3}, 125 \mathrm{MHz}\right): \delta$ (major isomer) $138.8(\mathrm{C} 2), 134.5$ (C5), 134.2 (C6), 127.1 (C1), 118.1 (nitrile C7), 115.8 (C4), 85.0 (C8), 24.7 (C9); ${ }^{11} \mathrm{~B}$ NMR $\left(\mathrm{CDCl}_{3}, 96 \mathrm{MHz}\right): \delta$ 29.92; FT-IR (neat): 2980, 2228, 1582, 1551, 1480, 1416, 1399, 1373, 1335, 1271, 1144, 1084, 1069, 963, 862, 841, 763, $673 \mathrm{~cm}^{-1}$; LRMS (\% rel. int.): $\mathrm{m} / \mathrm{e}$ (major isomer) $307 \mathrm{M}^{+}$(16), 292 (51), 266 (100), 251 (45), 228 (17), 170 (15); Anal. Calcd for $\mathrm{C}_{13} \mathrm{H}_{15} \mathrm{BBrNO}_{2}$ : C, 50.70; H, 4.91; N, 4.55. Found: C, 50.29; H, 4.75; N, 4.57. HRMS Calcd for $\mathrm{C}_{13} \mathrm{H}_{15} \mathrm{BBrNO}_{2}$ : 307.0379. Found: 307.0376 . 
Table 1, Entry 4: Borylation of 4-Iodobenzonitrile.

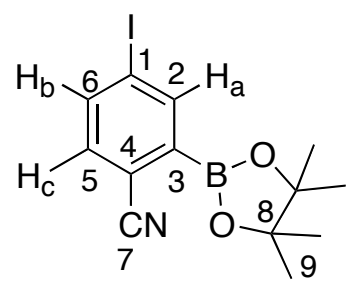

General procedure B was applied to 4-iodobenzonitrile (229.1 mg, $1 \mathrm{mmol}, 1$ equivalent) and $\mathrm{B}_{2} \mathrm{Pin}_{2}(254.0 \mathrm{mg}, 1 \mathrm{mmol}, 2$ equivalents of boron) with a reaction time of 40h. Only one isomer was observed in crude reaction mixture by GC and by ${ }^{1} \mathrm{H} \mathrm{NMR}$ spectroscopy. Solvent was removed under reduced pressure, and the crude mixture was eluted with $\mathrm{CH}_{2} \mathrm{Cl}_{2}$ through a plug of silica gel to afford the single pure isomer $(255 \mathrm{mg}$, $71 \%$ ) as a white solid, $\mathrm{mp} 77-79^{\circ} \mathrm{C}$. gHMBC spectroscopy (Figure 3) was used to assign the single isomer as 4-iodo-2-(4,4,5,5-tetramethyl-1,3,2-dioxaboryl)benzonitrile. ${ }^{1} \mathrm{H}$ NMR $\left(\mathrm{CDCl}_{3}, 500 \mathrm{MHz}\right): \delta 8.19\left(\mathrm{~d}, J=2.0 \mathrm{~Hz}, 1 \mathrm{H}, \mathrm{H}_{\mathrm{a}}\right), 7.85(\mathrm{dd}, J=8.3,2.0 \mathrm{~Hz}, 1 \mathrm{H}$, $\left.\mathrm{H}_{\mathrm{b}}\right), 7.36\left(\mathrm{~d}, J=8.3 \mathrm{~Hz}, 1 \mathrm{H}, \mathrm{H}_{\mathrm{c}}\right), 1.34$ (br s, $\left.12 \mathrm{H}\right) ;{ }^{13} \mathrm{C} \mathrm{NMR}\left\{{ }^{1} \mathrm{H}\right\}\left(\mathrm{CDCl}_{3}, 125 \mathrm{MHz}\right): \delta$ 144.6 (C2), 140.1 (C6), 134.3 (C5), 118.3 (nitrile C7), 116.3 (C4), 99.8 (C1), 85.1 (C8), 24.7 (C9); ${ }^{11} \mathrm{~B}$ NMR $\left(\mathrm{CDCl}_{3}, 96 \mathrm{MHz}\right): \delta$ 29.77; FT-IR (neat): 2980, 2228, 1576, 1545 , $1480,1414,1395,1374,1335,1271,1140,1071,963,859,839,826,673 \mathrm{~cm}^{-1}$; LRMS (\% rel. int.): m/e $355 \mathrm{M}^{+}$(42), 340 (75), 314 (100), 297 (72), 256 (77), 228 (11); Anal. Calcd for $\mathrm{C}_{13} \mathrm{H}_{15} \mathrm{BINO}_{2}$ : C, 43.99; H, 4.26; N, 3.95. Found: C, 44.17; H, 4.44; N, 3.88.

Table 1, Entry 5: Borylation of 4-methylbenzonitrile.
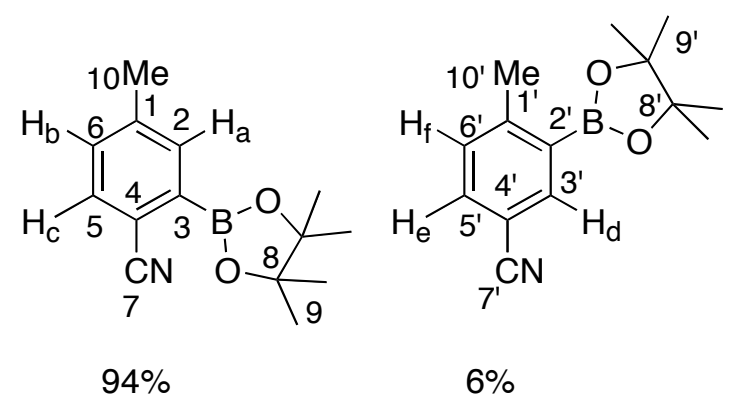

General procedure A was applied to 4-methylbenzonitrile (468.6 mg, $4 \mathrm{mmol}$ ) and HBPin (145 $\mu \mathrm{L}, 128 \mathrm{mg}, 1 \mathrm{mmol})$ using $6 \mathrm{~mol} \%$ [Ir] with a reaction time of $72 \mathrm{~h}$. The 
ratio of the two isomers in the crude reaction mixture by GC was 94:6. Kugelrohr distillation furnished a mixture of the two isomeric borylated products $(156 \mathrm{mg}, 64 \%)$ as colorless oil which solidified on standing. The ratio of the two isomers in the isolated product by GC was 92:8. The major isomer was assigned as 4-methyl-2-(4,4,5,5tetramethyl-1,3,2-dioxaboryl)benzonitrile by the NOESY1D spectrum and by preparing an authentic sample using a slightly modified literature procedure. ${ }^{4}{ }^{1} \mathrm{H} \mathrm{NMR}\left(\mathrm{CDCl}_{3}\right.$, $500 \mathrm{MHz}$ ): $\delta$ (major isomer) $7.67\left(\right.$ br s, $\left.1 \mathrm{H}, \mathrm{H}_{\mathrm{a}}\right), 7.57\left(\mathrm{~d}, J=7.9 \mathrm{~Hz}, 1 \mathrm{H}, \mathrm{H}_{\mathrm{b}}\right.$ or $\mathrm{H}_{\mathrm{c}}$ ), 7.30 $\left(\mathrm{d}, J=7.9 \mathrm{~Hz}, 1 \mathrm{H}, \mathrm{H}_{\mathrm{b}}\right.$ or $\left.\mathrm{H}_{\mathrm{c}}\right), 2.38(\mathrm{~s}, 3 \mathrm{H}), 1.36($ br s, $12 \mathrm{H}) ;{ }^{13} \mathrm{C} \mathrm{NMR}\left\{{ }^{1} \mathrm{H}\right\}\left(\mathrm{CDCl}_{3}, 125\right.$ MHz): $\delta$ (major isomer) 142.2 (C1), 136.5, 133.4, 131.8 (C2, C5 and C6), 119.2 (nitrile C7), 114.2 (C4), 84.7 (C8), 24.8 (C9), 21.5 (C10); ${ }^{11} \mathrm{~B}$ NMR ( $\left.\mathrm{CDCl}_{3}, 96 \mathrm{MHz}\right)$ : $\delta$ 30.36; FT-IR (neat): 2980, 2932, 2226, 1603, 1491, 1447, 1408, 1391, 1381, 1373, 1346, 1265 , 1213, 1140, 1069, 965, 853, 828, 675, $661 \mathrm{~cm}^{-1}$; LRMS (\% rel. int.): m/e $243 \mathrm{M}^{+}$(46), 228 (70), 202 (100), 185 (52), 144 (92), 117 (25); Anal. Calcd for $\mathrm{C}_{14} \mathrm{H}_{18} \mathrm{BNO}_{2}$ : C, 69.17; $\mathrm{H}, 7.46 ; \mathrm{N}$, 5.76. Found: C, 68.74; H, 7.64; N, 5.62. HRMS Calcd for $\mathrm{C}_{14} \mathrm{H}_{18} \mathrm{BNO}_{2}$ : 243.1431. Found: 243.1425.

\section{Preparation of an authentic sample of 4-methyl-2-(4,4,5,5-tetramethyl-1,3,2- dioxaboryl)-benzonitrile:}

In a glove box, a $100 \mathrm{~mL}$ schlenk flask, equipped with a magnetic stirring bar, was charged with $\mathrm{Pd}_{2}(\mathrm{dba})_{3} \quad\left(\begin{array}{llllll}13.8 & \mathrm{mg}, & 0.015 & \mathrm{mmol}, & 3 \mathrm{~mol} \% & \mathrm{Pd}\end{array}\right)$ and tricyclohexylphosphine $\left(\mathrm{PCy}_{3}, 20.2 \mathrm{mg}, 0.072 \mathrm{mmol}, 7.2 \mathrm{~mol} \%\right)$. Dioxane $(6 \mathrm{~mL})$ was added and the resulting mixture was stirred for 30 minutes at room temperature. $\mathrm{B}_{2} \mathrm{Pin}_{2}$ (280 mg, $1.1 \mathrm{mmol}), \mathrm{KOAc}(147 \mathrm{mg}, 1.5 \mathrm{mmol})$, and 2-bromo-4-methylbenzonitrile (196 mg, $1 \mathrm{mmol}$ ) were added successively. The schlenk flask was brought to a schlenk line. A condenser was attached, and the flask was flushed with nitrogen. The reaction mixture was stirred at $80^{\circ} \mathrm{C}$ for $12 \mathrm{~h}$. The mixture was treated with water $(5 \mathrm{~mL})$, and the product was extracted with ether, washed with brine, and dried over $\mathrm{MgSO}_{4}$. Kugelrohr distillation furnished the desired product $(151 \mathrm{mg}, 62 \%)$ as a colorless oil. Its spectral data matched the major isomer obtained from the catalytic borylation of 4-methylbenzonitrile described earlier. 
Table 1, Entry 6: Borylation of 4-methoxylbenzonitrile.
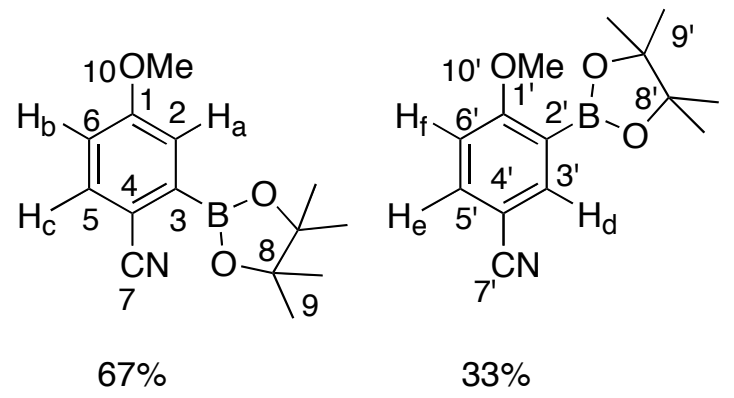

General procedure A was applied to 4-methoxybenzonitrile (266.3 mg, $2 \mathrm{mmol})$ and HBPin $(73 \mu \mathrm{L}, 64 \mathrm{mg}, 0.5 \mathrm{mmol})$ with a reaction time of $24 \mathrm{~h}$. The ratio of the two isomers in the crude reaction mixture by ${ }^{1} \mathrm{H}$ NMR spectroscopy was 67:33. Kugelrohr distillation furnished a mixture of the two isomeric borylated products ( $84 \mathrm{mg}, 65 \%)$ as a colorless oil. The ratio of the two isomers in the isolated product by ${ }^{1} \mathrm{H}$ NMR spectroscopy was 67:33. The NOESY1D and gHMBC spectra (Figure 4) were used to assign the major isomer as 4-methoxy-2-(4,4,5,5-tetramethyl-1,3,2dioxaboryl)benzonitrile. ${ }^{1} \mathrm{H}$ NMR $\left(\mathrm{CDCl}_{3}, 500 \mathrm{MHz}\right): \delta$ (major isomer) $7.59(\mathrm{~d}, J=8.5$ $\left.\mathrm{Hz}, 1 \mathrm{H}, \mathrm{H}_{\mathrm{c}}\right), 7.31\left(\mathrm{~d}, J=2.9 \mathrm{~Hz}, 1 \mathrm{H}, \mathrm{H}_{\mathrm{a}}\right), 6.97\left(\mathrm{dd}, J=8.5,2.9 \mathrm{~Hz}, 1 \mathrm{H}, \mathrm{H}_{\mathrm{b}}\right), 3.84(\mathrm{~s}, 3 \mathrm{H})$, 1.35 (br s, 12H), (minor isomer) $7.93\left(\mathrm{~d}, J=2.4 \mathrm{~Hz}, 1 \mathrm{H}, \mathrm{H}_{\mathrm{d}}\right), 7.65$ (dd, $J=8.8,2.4 \mathrm{~Hz}$, $\left.1 \mathrm{H}, \mathrm{H}_{\mathrm{e}}\right), 6.87\left(\mathrm{~d}, J=8.8 \mathrm{~Hz}, 1 \mathrm{H}, \mathrm{H}_{\mathrm{f}}\right), 3.85(\mathrm{~s}, 3 \mathrm{H}), 1.32\left(\right.$ br s, 12H); ${ }^{13} \mathrm{C} \mathrm{NMR}\left\{{ }^{1} \mathrm{H}\right\}$ $\left(\mathrm{CDCl}_{3}, 125 \mathrm{MHz}\right): \delta$ (major isomer) 161.7 (C1), 135.3 (C5), 120.6 (C2), 119.3 (nitrile C7), 117.0 (C6), 108.8 (C4), 84.8 (C8), 55.5 (C10), 24.73 (C9), (minor isomer) 166.9 (C1' ), 140.9 (C3' ), 136.6 (C5' ), 119.2 (nitrile C7' ), 110.7 (C6' ), 103.6 (C4' ), 84.1 (C8' ), $55.5\left(\mathrm{C} 10^{\prime}\right), 24.7\left(\mathrm{C}^{\prime}\right) ;{ }^{11} \mathrm{~B} \mathrm{NMR}\left(\mathrm{CDCl}_{3}, 96 \mathrm{MHz}\right): \delta$ 30.50; FT-IR (neat): 2980, 2942, 2842, 2224, 1601, 1493, 1466, 1449, 1424, 1412, 1373, 1345, 1271, 1238, 1144, 1060, 1030, 965, 853, $830 \mathrm{~cm}^{-1}$; LRMS (\% rel. int.): m/e $259 \mathrm{M}^{+}$(100), 244 (61), 232 (9), 216 (73), 201 (65), 186 (25), 174 (20), 160 (79); HRMS Calcd for $\mathrm{C}_{14} \mathrm{H}_{18} \mathrm{BNO}_{3}$ : 259.1380. Found: 259.1383. 
Table 1, Entry 7: Borylation of 4-thiometylbenzonitrile.
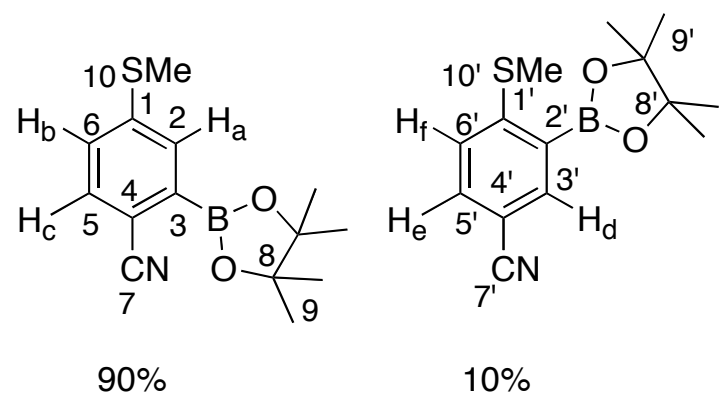

General procedure A was applied to 4-thiomethylbenzonitrile (298.5 mg, $2 \mathrm{mmol}$, 2 equivalents) and $\mathrm{B}_{2} \mathrm{Pin}_{2}\left(127 \mathrm{mg}, 0.5 \mathrm{mmol}, 1\right.$ equivalent of boron) at $80{ }^{\circ} \mathrm{C}$ with a reaction time of $18 \mathrm{~h}$. The ratio of the two isomers in the crude reaction mixture by ${ }^{1} \mathrm{H}$ NMR spectroscopy was 90:10. Kugelrohr distillation gave a fraction (155 mg) containing two isomers along with small amount of $\mathrm{d}^{t}$ bpy. Passing a $\mathrm{CH}_{2} \mathrm{Cl}_{2}$ solution of that fraction through a plug of silica furnished a mixture of two isomeric borylated products $(150 \mathrm{mg}$, $55 \%$ ) as a white solid. The ratio of the two isomers in the isolated product by ${ }^{1} \mathrm{H}$ NMR spectroscopy was $87: 13$. The NOESY1D and gHMBC spectra (Figure 5) were used to assign the major isomer as 4-thiomethyl-2-(4,4,5,5-tetramethyl-1,3,2dioxaboryl)benzonitrile. ${ }^{1} \mathrm{H}$ NMR $\left(\mathrm{CDCl}_{3}, 500 \mathrm{MHz}\right): \delta$ (major isomer) $7.63(\mathrm{~d}, J=2.0$ $\left.\mathrm{Hz}, 1 \mathrm{H}, \mathrm{H}_{\mathrm{a}}\right), 7.54\left(\mathrm{~d}, J=8.3 \mathrm{~Hz}, 1 \mathrm{H}, \mathrm{H}_{\mathrm{c}}\right), 7.27$ (dd, $\left.J=8.3,2.0 \mathrm{~Hz}, 1 \mathrm{H}, \mathrm{H}_{\mathrm{b}}\right), 2.48(\mathrm{~s}, 3 \mathrm{H})$, 1.35 (br s, 12H), (minor isomer) $7.92\left(\mathrm{~d}, J=2.0 \mathrm{~Hz}, 1 \mathrm{H}, \mathrm{H}_{\mathrm{d}}\right), 7.56(\mathrm{dd}, J=8.3,2.0 \mathrm{~Hz}$, $\left.1 \mathrm{H}, \mathrm{H}_{\mathrm{e}}\right), 7.14\left(\mathrm{~d}, J=8.3 \mathrm{~Hz}, 1 \mathrm{H}, \mathrm{H}_{\mathrm{f}}\right), 2.44(\mathrm{~s}, 3 \mathrm{H}), 1.34($ br s, $12 \mathrm{H}) ;{ }^{13} \mathrm{C} \mathrm{NMR}\left\{{ }^{1} \mathrm{H}\right\}$ $\left(\mathrm{CDCl}_{3}, 125 \mathrm{MHz}\right): \delta$ (major isomer) 144.7 (C1), 133.4 (C5), 132.2 (C2), 127.1 (C6), 119.1 (nitrile C7), 112.4 (C4), 84.8 (C8), 24.7 (C9), 14.61 (C10), (minor isomer) 152.7 (C1' ), 139.3 (C3' ), 134.1 (C5' ), 122.9 (C6' ), 119.0 (nitrile C7' ), 106.7 (C4' ), 84.6 (C8' ), 24.7 (C9' ), 15.0 (C10' ); ${ }^{11} \mathrm{~B}$ NMR ( $\left.\mathrm{CDCl}_{3}, 96 \mathrm{MHz}\right): \delta$ 30.27; FT-IR (neat): 2980, 2928, 2224, 1584, 1547, 1483, 1397, 1381, 1373, 1345, 1269, 1213, 1167, 1142, 1107 , 1059, 963, 871, 847, 825, 769, 741, $669 \mathrm{~cm}^{-1}$; LRMS (\% rel. int.): m/e $275 \mathrm{M}^{+}$(100), 260 (26), 232 (41), 217 (46), 202 (9), 190 (10), 175 (54); Anal. Calcd for $\mathrm{C}_{14} \mathrm{H}_{18} \mathrm{BNO}_{2} \mathrm{~S}$ : C, 61.11; H, 6.59; N, 5.09. Found: C, 61.24; H, 6.95; N, 5.05; HRMS Calcd for $\mathrm{C}_{14} \mathrm{H}_{18} \mathrm{BNO}_{2} \mathrm{~S}: 275.1151$. Found: 275.1157. 
Table 1, Entry 8: Borylation of 4-dimethylaminobenzonitrile.

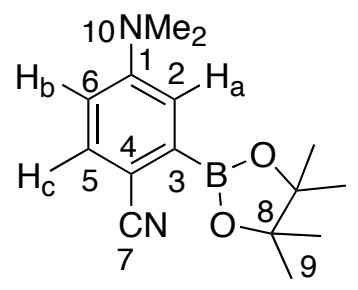

General procedure B was applied to 4-dimethylaminobenzonitrile (146.2 mg, 1 mmol, 1 equivalent) and $\mathrm{B}_{2} \operatorname{Pin}_{2}(254.0 \mathrm{mg}, 1 \mathrm{mmol}, 2$ equivalents of boron) using 6 $\mathrm{mol} \%$ [Ir] with a reaction time of $72 \mathrm{~h}$. Only one isomer was observed in the crude reaction mixture by GC and by ${ }^{1} \mathrm{H}$ NMR spectroscopy. Solvent was removed under reduced pressure, and the crude mixture was eluted with $\mathrm{CH}_{2} \mathrm{Cl}_{2}$ through a plug of silica gel to afford the single pure isomer $(180 \mathrm{mg}, 66 \%)$ as a white solid, mp $110-11{ }^{\circ} \mathrm{C}$. The NOESY1D and gHMBC spectra (Figure 6) were used to assign the single isomer as 4-dimethylamino-2-(4,4,5,5-tetramethyl-1,3,2-dioxaboryl)benzonitrile. ${ }^{1} \mathrm{H}$ NMR $\left(\mathrm{CDCl}_{3}\right.$, $300 \mathrm{MHz}): \delta 7.48\left(\mathrm{~d}, J=8.8 \mathrm{~Hz}, 1 \mathrm{H}, \mathrm{H}_{\mathrm{c}}\right), 7.04\left(\mathrm{~d}, J=2.9 \mathrm{~Hz}, 1 \mathrm{H}, \mathrm{H}_{\mathrm{a}}\right), 6.67(\mathrm{dd}, J=8.8$,

$\left.2.9 \mathrm{~Hz}, 1 \mathrm{H}, \mathrm{H}_{\mathrm{b}}\right), 3.01(\mathrm{~s}, 6 \mathrm{H}), 1.35$ (br s, $\left.12 \mathrm{H}\right) ;{ }^{13} \mathrm{C} \mathrm{NMR}\left\{{ }^{1} \mathrm{H}\right\}\left(\mathrm{CDCl}_{3}, 75 \mathrm{MHz}\right): \delta 151.4$ (C1), 134.8 (C5), 120.7 (nitrile C7), 118.1 (C2), 113.2 (C6), 102.3 (C4), 84.5 (C8), 39.9 (C10), 24.7 (C9); ${ }^{11} \mathrm{~B}$ NMR $\left(\mathrm{CDCl}_{3}, 96 \mathrm{MHz}\right): \delta$ 30.5; FT-IR (neat): 2980, 2932, 2815, 2213, 1597, 1553, 1508, 1485, 1429, 1416, 1374, 1337, 1271, 1230, 1169, 1144, 1053, 968, 847, $816 \mathrm{~cm}^{-1}$; LRMS (\% rel. int.): m/e $272 \mathrm{M}^{+}$(100), 257 (7), 229 (11), 214 (11), 189 (6), 173 (23); Anal. Calcd for $\mathrm{C}_{15} \mathrm{H}_{21} \mathrm{BN}_{2} \mathrm{O}_{2}: \mathrm{C}, 66.2 ; \mathrm{H}, 7.78 ; \mathrm{N}, 10.29$. Found: C, $66.54 ; \mathrm{H}, 7.76 ; \mathrm{N}, 10.06$.

\section{Table 1, Entry 9: Borylation of methyl 4-cyanobenzoate.}

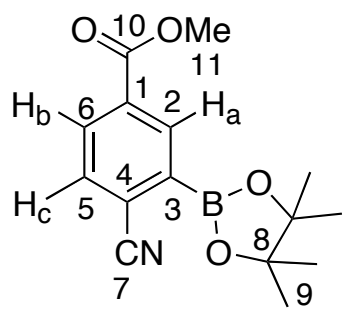

General procedure B was applied to methyl 4-cyanobenzoate (161.2 mg, $1 \mathrm{mmol}$, 1 equivalent) and $\mathrm{B}_{2} \mathrm{Pin}_{2}(203.0 \mathrm{mg}, 0.8 \mathrm{mmol}, 1.6$ equivalents of boron) with a reaction 
time of $48 \mathrm{~h}$. Only one isomer was observed in the crude reaction mixture by GC and by ${ }^{1} \mathrm{H}$ NMR spectroscopy. Solvent was removed under reduced pressure, and the crude mixture was eluted with $\mathrm{CH}_{2} \mathrm{Cl}_{2}$ through a plug of silica gel to afford the single pure isomer (190 $\mathrm{mg}, 66 \%)$ as a white solid, $\mathrm{mp} 136-137{ }^{\circ} \mathrm{C}$. The NOESY1D and gHMBC spectra (Figure 7) were used to assign the single isomer as methyl 4-cyano-3-(4,4,5,5tetramethyl-1,3,2-dioxaboryl)benzoate. ${ }^{1} \mathrm{H}$ NMR $\left(\mathrm{CDCl}_{3}, 300 \mathrm{MHz}\right): \delta 8.49$ (d, $J=1.7$ $\mathrm{Hz}, 1 \mathrm{H}, \mathrm{H}_{\mathrm{a}}$ ), 8.14 (dd, $\left.J=8.1,1.9 \mathrm{~Hz}, 1 \mathrm{H}, \mathrm{H}_{\mathrm{b}}\right), 7.75$ (d, $J=8.1 \mathrm{~Hz}, 1 \mathrm{H}, \mathrm{H}_{\mathrm{c}}$ ), 3.93 (s, $3 \mathrm{H}$ ), 1.37 (br s, $12 \mathrm{H}) ;{ }^{13} \mathrm{C}$ NMR $\left\{{ }^{1} \mathrm{H}\right\}\left(\mathrm{CDCl}_{3}, 75 \mathrm{MHz}\right): \delta 165.6$ (C10), $136.6(\mathrm{C} 2), 133.4$ (C5), 132.7 (C1), 131.9 (C6), 121.1 (C4), 118.1 (nitrile C7), 85.1 (C8), 52.6 (C11), 24.8 (C9); ${ }^{11} \mathrm{~B}$ NMR (CDCl, $\left.96 \mathrm{MHz}\right): \delta 30.1$; FT-IR (neat): 2980, 2954, 2230, 1721, 1603 , $1487,1410,1375,1337,1279,1251,1144,1115,1069,976,851,770,654 \mathrm{~cm}^{-1}$; LRMS (\% rel. int.): m/e $287 \mathrm{M}^{+}$(5), 272 (32), 256 (20), 244 (100), 229 (18), 188 (26), 156 (14); Anal. Calcd for $\mathrm{C}_{15} \mathrm{H}_{18} \mathrm{BNO}_{4}$ : C, 62.75; H, 6.32; N, 4.88. Found: C, 62.33; H, 6.26; N, 4.79. HRMS Calcd for $\mathrm{C}_{15} \mathrm{H}_{18} \mathrm{BNO}_{4}: 287.1329$. Found: 287.1327 .

\section{Table 1, Entry 10: Borylation of 4'-cyanoacetanilide.}

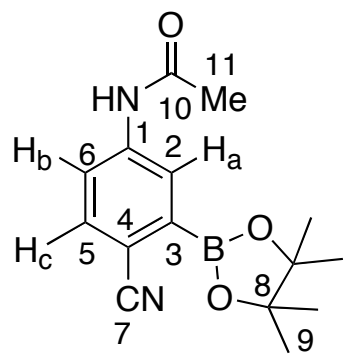

General procedure B was applied to 4'-cyanoacetanilide (160.2 $\mathrm{mg}, 1 \mathrm{mmol}, 1$ equivalent) and $\mathrm{B}_{2} \mathrm{Pin}_{2}$ (406 mg, $1.6 \mathrm{mmol}, 3.2$ equivalents of boron) using $8 \mathrm{~mol} \%$ [Ir] with a reaction time of $18 \mathrm{~h}$. One borylated isomer was observed in the crude reaction mixture by $\mathrm{GC}$ and by ${ }^{1} \mathrm{H}$ NMR spectroscopy along with a small amount of borylated/reduced (reduction of carbonyl group to $\mathrm{CH}_{2}$ ) as a side product. Solvent was removed under reduced pressure. Column chromatography (ether, $\mathrm{Rf}=0.5$ ) gave a mixture of the desired product and pinacol $(239 \mathrm{mg})$. Kugelrohr distillation furnished the desired product (177 $\mathrm{mg}, 62 \%$ ) as a white solid, $\mathrm{mp} 178-180{ }^{\circ} \mathrm{C}$. The gHMBC spectrum (Figure 8) was used to assign the single isomer as 4'-cyano-3'-(4,4,5,5-tetramethyl-1,3,2- 
dioxaboryl)acetanilide. ${ }^{1} \mathrm{H}$ NMR $\left(\mathrm{CDCl}_{3}, 500 \mathrm{MHz}\right): \delta 7.99\left(\mathrm{dd}, J=8.3,2.0 \mathrm{~Hz}, 1 \mathrm{H}, \mathrm{H}_{\mathrm{b}}\right)$, 7.85 (s, $1 \mathrm{H}, \mathrm{N}-\mathrm{H}), 7.77$ (d, $\left.J=2.4 \mathrm{~Hz}, 1 \mathrm{H}, \mathrm{H}_{\mathrm{a}}\right), 7.63$ (d, $\left.J=8.3 \mathrm{~Hz}, 1 \mathrm{H}, \mathrm{H}_{\mathrm{c}}\right), 2.18(\mathrm{~s}, 3 \mathrm{H})$, 1.32 (br s, $12 \mathrm{H}) ;{ }^{13} \mathrm{C}$ NMR $\left\{{ }^{1} \mathrm{H}\right\}\left(\mathrm{CDCl}_{3}, 75 \mathrm{MHz}\right): \delta 168.8$ (C10), 141.1 (C1), 134.7 (C5), 125.9 (C2), 121.5 (C6), 119 (nitrile C7), 111.6 (C4), 84.8 (C8), 24.7 (C9), 24.6 (C11); ${ }^{11} \mathrm{~B}$ NMR (CDCl 3 , $\left.96 \mathrm{MHz}\right): \delta$ 30.52; FT-IR (neat): 3319, 3104, 2980, 2934, 2225 , 1701, 1678, 1601, 1578, 1532, 1497, 1416, 1373, 1344, 1302, 1258, 1140, 1063, 965, 853, 800, 743, $675 \mathrm{~cm}^{-1}$; LRMS (\% rel. int.): m/e $286 \mathrm{M}^{+}$(100), 271 (18), 253 (32), 244 (89), 228 (68), 201 (42), 187 (68), 158 (13), 144 (58); HRMS Calcd for $\mathrm{C}_{15} \mathrm{H}_{19} \mathrm{BN}_{2} \mathrm{O}_{3}$ : 286.1489. Found: 286.1493.

Table 1, Entry 11: Borylation of 4-(trifluoromethyl)benzonitrile.

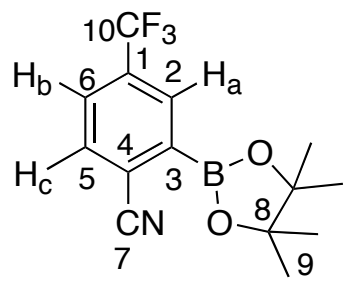

General procedure B was applied to 4-(trifluoromethyl)benzonitrile (171.1 mg, 1 mmol, 1 equivalent) and HBPin ( $175 \mu \mathrm{L}, 154 \mathrm{mg}, 1.2 \mathrm{mmol})$ in $n$-hexane $(3 \mathrm{~mL})$ with a reaction time of $12 \mathrm{~h}$. One monoborylated product and one diborylated product were observed in the crude reaction mixture by GC (90:10). Solvent was removed under reduced pressure. The crude mixture was eluted with $\mathrm{CH}_{2} \mathrm{Cl}_{2}$ through a plug of silica gel. Sublimation furnished the desired single monoborylated product (203 $\mathrm{mg}, 68 \%$ ) as a white solid, mp 79-80 ${ }^{\circ} \mathrm{C} .{ }^{13} \mathrm{C}$ NMR spectroscopy was used to assign the single isomer as 4-(trifluoromethyl)-2-(4,4,5,5-tetramethyl-1,3,2-dioxaboryl)benzonitrile. $\quad{ }^{1} \mathrm{H} \quad$ NMR $\left(\mathrm{CDCl}_{3}, 500 \mathrm{MHz}\right): \delta 8.12\left(\mathrm{~s}, 1 \mathrm{H}, \mathrm{H}_{\mathrm{a}}\right), 7.81\left(\mathrm{~d}, J=7.8 \mathrm{~Hz}, 1 \mathrm{H}, \mathrm{H}_{\mathrm{b}}\right.$ or $\left.\mathrm{H}_{\mathrm{c}}\right), 7.76(\mathrm{~d}, J=7.8$ $\mathrm{Hz}, 1 \mathrm{H}, \mathrm{H}_{\mathrm{b}}$ or $\left.\mathrm{H}_{\mathrm{c}}\right), 1.38$ (br s, $\left.12 \mathrm{H}\right) ;{ }^{13} \mathrm{C}$ NMR $\left\{{ }^{1} \mathrm{H}\right\}\left(\mathrm{CDCl}_{3}, 125 \mathrm{MHz}\right): \delta 133.7$ (C5), $133.3\left(\mathrm{q},{ }^{2} J_{\mathrm{C}-\mathrm{F}}=33.2 \mathrm{~Hz}, \mathrm{C} 1\right), 132.5\left(\mathrm{q},{ }^{3} J_{\mathrm{C}-\mathrm{F}}=2.1 \mathrm{~Hz}, \mathrm{C} 2\right), 127.8\left(\mathrm{q},{ }^{3} J_{\mathrm{C}-\mathrm{F}}=3.3 \mathrm{~Hz}, \mathrm{C} 6\right)$, 122.1 (q, ${ }^{1} J_{\mathrm{C}-\mathrm{F}}=273 \mathrm{~Hz}, \mathrm{C} 10$ ), 120.7 (C4), 117.6 (nitrile C7), 85.3 (C8), 24.7 (C9); ${ }^{11} \mathrm{~B}$ NMR (CDCl $3,96 \mathrm{MHz}): \delta 30.03 ;{ }^{19} \mathrm{~F}$ NMR $\left(\mathrm{CDCl}_{3}, 282 \mathrm{MHz}\right): \delta$-63.4; FT-IR (neat): 2982, 2234, 1613, 1574, 1423, 1354, 1306, 1271, 1175, 1142, 1082, 1065, 965, 878, 849, 
$675 \mathrm{~cm}^{-1}$; LRMS (\% rel. int.): m/e $297 \mathrm{M}^{+}$(23), 282 (100), 256 (81), 239 (27), 198 (19), 171 (10); HRMS Calcd for $\mathrm{C}_{14} \mathrm{H}_{15} \mathrm{BF}_{3} \mathrm{NO}_{2}: 297.1148$. Found: 297.1144.

\section{Borylation of benzonitrile.}

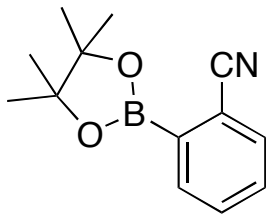

$5.7 \%$

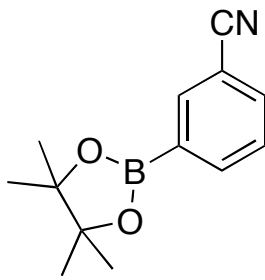

$64.4 \%$

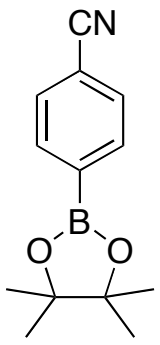

$29.9 \%$

General procedure A was applied to benzonitrile (412.5 $\mathrm{mg}, 4 \mathrm{mmol}$ ) and HBPin $(145 \mu \mathrm{L}, 128 \mathrm{mg}, 1 \mathrm{mmol})$ with a reaction time of $12 \mathrm{~h}$. The ratio of the three isomers in the crude reaction mixture by GC was 5.7:64.4:29.9. Solvent and excess substrate were removed under reduced pressure. The crude mixture was eluted with $\mathrm{CH}_{2} \mathrm{Cl}_{2}$ through a plug of silica gel to afford a mixture of the three isomeric borylated products $(176 \mathrm{mg}$, $77 \%$ ) as a white solid. ${ }^{1} \mathrm{H}$ NMR, gCOSY, and gHMBC spectroscopy were used to assign the major isomer as 3-(4,4,5,5-tetramethyl-1,3,2-dioxaboryl)benzonitrile. ${ }^{1} \mathrm{H}$ NMR $\left(\mathrm{CDCl}_{3}, 300 \mathrm{MHz}\right): \delta$ (major/meta isomer) 8.07-8.05 (br s, $\left.1 \mathrm{H}\right), 7.98$ (td, $J=7.5,1.2 \mathrm{~Hz}$, $1 \mathrm{H}), 7.71-7.67(\mathrm{~m}, 1 \mathrm{H}), 7.44(\mathrm{dt}, J=7.5,0.7 \mathrm{~Hz}, 1 \mathrm{H}), 1.32$ (br s, 12H), (minor/para isomer) 7.87-7.84 (m, 2H), 7.62-7.59 (m, 2H), 1.32 (br s, $12 \mathrm{H}) ;{ }^{13} \mathrm{C} \mathrm{NMR}\left\{{ }^{1} \mathrm{H}\right\}\left(\mathrm{CDCl}_{3}\right.$, $75 \mathrm{MHz}$ ): $\delta$ (major/meta isomer) 138.7, 138.3, 134.3, 128.3, 118.7, 112, 84.4, 24.8, (minor/para isomer) 135, 131, 118.7, 114.4, 84.7, 24.8; ${ }^{11} \mathrm{~B} \mathrm{NMR}\left(\mathrm{CDCl}_{3}, 96 \mathrm{MHz}\right): \delta$ 31.2; FT-IR (neat): 3063, 2980, 2934, 2230, 1603, 1483, 1398, 1360, 1329, 1271, 1143, 1088, 964, 880, 849, 700, $653 \mathrm{~cm}^{-1}$; LRMS (\% rel. int.): m/e (major/meta isomer) $229 \mathrm{M}^{+}$ (11), $230 \mathrm{M}^{+1}$ (29), 214 (100), 186 (10), 143 (44), (minor/para isomer) $229 \mathrm{M}^{+}$(5), 230 $\mathrm{M}^{+1}$ (14), 186 (9), 143 (41); HRMS Calcd for $\mathrm{C}_{13} \mathrm{H}_{16} \mathrm{BNO}_{2}$ : 229.1274. Found: 229.1271. 


\section{Borylation of anisole.}

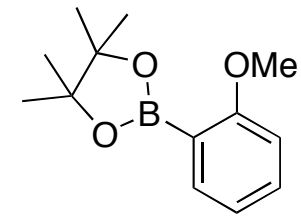

$2.1 \%$

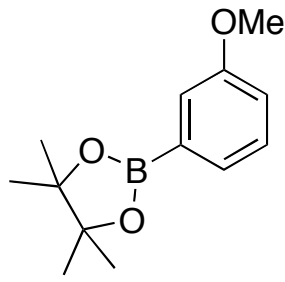

$78.9 \%$

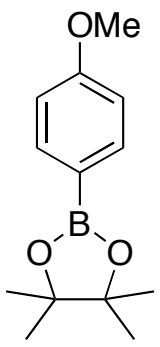

$19 \%$

General procedure A was applied to anisole (432.6 mg, $4 \mathrm{mmol}$ ) and HBPin (145 $\mu \mathrm{L}, 128 \mathrm{mg}, 1 \mathrm{mmol}$ ) with a reaction time of $24 \mathrm{~h}$. The ratio of the three isomers in the crude reaction mixture by GC was 2.1:78.9:19. Solvent and excess substrate were removed under reduced pressure. The crude mixture was eluted with $\mathrm{CH}_{2} \mathrm{Cl}_{2}$ through a plug of silica gel to afford a mixture of the three isomeric borylated products $(193 \mathrm{mg}$, $82 \%$ ) as a colorless oil. ${ }^{1} \mathrm{H}$ NMR spectroscopy was used to assign the major isomer as 3-(4,4,5,5-tetramethyl-1,3,2-dioxaboryl)anisole. ${ }^{1} \mathrm{H} \quad \mathrm{NMR} \quad\left(\mathrm{CDCl}_{3}, 300 \mathrm{MHz}\right): \delta$ (major/meta isomer) 7.40-7.25 (m, 3H), $7.00(\mathrm{~m}, 1 \mathrm{H}), 3.82(\mathrm{~s}, 3 \mathrm{H}), 1.33$ (br s, 12H), (minor/para isomer) 7.75-7.72 (m, 2H), 6.89-6.86 (m, 2H), $3.81(\mathrm{~s}, 3 \mathrm{H}), 1.32(\mathrm{br} \mathrm{s}, 12 \mathrm{H})$. The spectra were in agreement with those described in the literature.

\section{Borylation of 1,3-dicyanobenzene.}

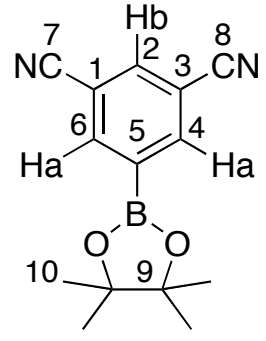

$74 \%$

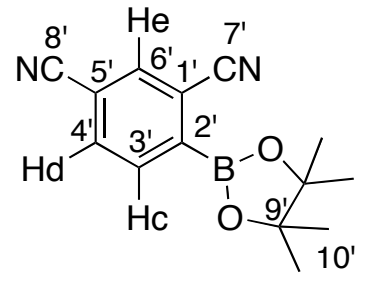

$26 \%$

General procedure A was applied to 1,3-dicyanobenzene (256.3 $\mathrm{mg}, 2 \mathrm{mmol})$ and HBPin $(73 \mu \mathrm{L}, 64 \mathrm{mg}, 0.5 \mathrm{mmol})$ with a reaction time of $12 \mathrm{~h}$. The ratio of the two isomers in the crude reaction mixture by ${ }^{1} \mathrm{H}$ NMR spectroscopy was 74:26. Kugelrohr 
distillation furnished a mixture of the two isomeric borylated products ( $79 \mathrm{mg}, 62 \%$ ) as a white solid. The ratio of the two isomers in the isolated product by ${ }^{1} \mathrm{H}$ NMR spectroscopy was 77:23. ${ }^{1} \mathrm{H}$ NMR spectroscopy was used to assign the major isomer as 5-(4,4,5,5tetramethyl-1,3,2-dioxaboryl)-1,3-dicyanobenzene. ${ }^{1} \mathrm{H}$ NMR $\left(\mathrm{CDCl}_{3}, 300 \mathrm{MHz}\right): \delta$ (major isomer) $8.24\left(\mathrm{~d}, J=1.7 \mathrm{~Hz}, 2 \mathrm{H}, \mathrm{H}_{\mathrm{a}}\right), 7.96\left(\mathrm{t}, J=1.7 \mathrm{~Hz}, 1 \mathrm{H}, \mathrm{H}_{\mathrm{b}}\right), 1.33(\mathrm{br} \mathrm{s}, 12 \mathrm{H})$, (minor isomer) $7.99\left(\mathrm{~d}, J=7.8 \mathrm{~Hz}, 1 \mathrm{H}, \mathrm{H}_{\mathrm{c}}\right), 7.92\left(\mathrm{~d}, J=1.4 \mathrm{~Hz}, 1 \mathrm{H}, \mathrm{H}_{\mathrm{e}}\right), 7.80(\mathrm{dd}, J=$ 7.8, $\left.1.4 \mathrm{~Hz}, 1 \mathrm{H}, \mathrm{H}_{\mathrm{d}}\right), 1.36$ (br s, $\left.12 \mathrm{H}\right) ;{ }^{13} \mathrm{C} \mathrm{NMR}\left\{{ }^{1} \mathrm{H}\right\}\left(\mathrm{CDCl}_{3}, 125 \mathrm{MHz}\right): \delta$ (major, meta isomer) 141.8 (C4, C6), 137.1 (C2), 116.7 (nitrile C7, C8), 113.5 (C1, C3), 85.2 (C9), 24.8 (C10), (minor, ortho isomer) 136.6, 136.1, 134.4, (C3', C4', C6' ) 118.6, 116.8, 116.7, 115.3, (C1', C5', C7', C8' ), 85.5 (C9' ), $24.7\left({\mathrm{C} 10^{\prime}}^{\prime}\right) .{ }^{11} \mathrm{~B} \mathrm{NMR}\left(\mathrm{CDCl}_{3}, 96 \mathrm{MHz}\right)$ : $\delta 30.03$; FT-IR (neat): 3073, 2982, 2237, 1595, 1418, 1398, 1374, 1339, 1233, 1215, 1169, 1144, 1129, 1064, 966, 897, 849, $698 \mathrm{~cm}^{-1}$; LRMS (\% rel. int.): m/e $254 \mathrm{M}^{+}$(7), 239 (100), 211 (15), 196 (4), 168 (43), 155 (7); Anal. Calcd for $\mathrm{C}_{14} \mathrm{H}_{15} \mathrm{BN}_{2} \mathrm{O}_{2}$ : C, 66.18; H, 5.95; N, 11.02. Found: C, 66; H, 6.02; N, 10.81 .

\section{Borylation of 1,3-difluorobenzene.}

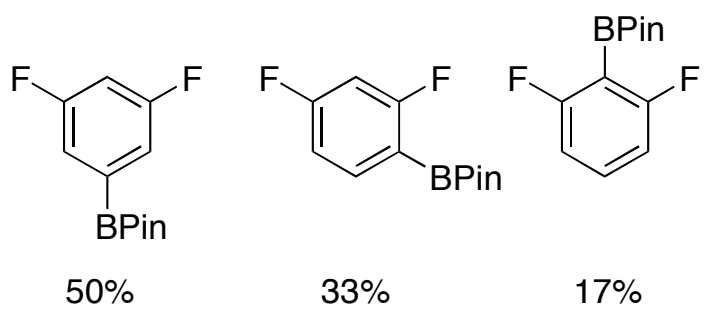

General procedure A was applied to 1,3-fluorobenzene $(228.2 \mathrm{mg}, 2 \mathrm{mmol})$ and HBPin $(73 \mu \mathrm{L}, 64 \mathrm{mg}, 0.5 \mathrm{mmol})$ with a reaction time of $1 \mathrm{~h}$. The ratio of the three isomers in the crude reaction mixture by GC-FID was 50:33:17, with GC-retention time of $7.92,8.17$, and 8.35 minutes respectively. ${ }^{13} \mathrm{C}$ NMR spectroscopy and authentic sample preparation were used for making regioisomeric assignments. Authentic samples of each isomer were synthesized using a slightly modified literature procedure. ${ }^{4}$

\section{Preparation of authentic samples of borylated 1,3-difluorobenzenes.}

In a glove box, a $100 \mathrm{~mL}$ schlenk flask, equipped with a magnetic stirring bar, was charged with $\mathrm{Pd}_{2}(\mathrm{dba})_{3} \quad\left(\begin{array}{lllllll}13.8 & \mathrm{mg}, & 0.015 & \mathrm{mmol}, & 3 & \mathrm{~mol} \% & \mathrm{Pd}\end{array}\right)$ and 
tricyclohexylphosphine ( $\left.\mathrm{PCy}_{3}, 20.2 \mathrm{mg}, 0.072 \mathrm{mmol}, 7.2 \mathrm{~mol} \%\right)$. Dioxane $(6 \mathrm{~mL})$ was added and the resulting mixture was stirred for 30 minutes at room temperature. $\mathrm{B}_{2} \mathrm{Pin}_{2}$ (280 $\mathrm{mg}, 1.1 \mathrm{mmol}$ ), KOAc (147 $\mathrm{mg}, 1.5 \mathrm{mmol}$ ), and the corresponding bromo substituted 1,3-difluorobenzene (193 mg, $1 \mathrm{mmol}$ ) were added successively. The schlenk flask was brought to a schlenk line. A condenser was attached, and the flask was flushed with nitrogen. The reaction mixture was stirred at $80^{\circ} \mathrm{C}$ for $12 \mathrm{~h}$. The mixture was treated with water $(5 \mathrm{~mL})$, and the product was extracted with ether, washed with brine, and dried over $\mathrm{MgSO}_{4}$. Crude material was eluted with $\mathrm{CH}_{2} \mathrm{Cl}_{2}$ through a plug of silica gel to afford the desired product. Characterization data for each isomer is described below.

\section{5-(4,4,5,5-tetramethyl-1,3,2-dioxaboryl)-1,3-fluorobenzene.}

$161 \mathrm{mg}$ (67\%); mp 47-48 ${ }^{\circ} \mathrm{C}$; GC-retention time 7.92 minute; ${ }^{1} \mathrm{H}$ NMR $\left(\mathrm{CDCl}_{3}\right.$, $300 \mathrm{MHz}): \delta 7.28-7.24(\mathrm{~m}, 2 \mathrm{H}), 6.85\left(\mathrm{tt},{ }^{2} J_{\mathrm{H}-\mathrm{F}}=9.1 \mathrm{~Hz},{ }^{4} J_{\mathrm{H}-\mathrm{H}}=2.5 \mathrm{H}, 1 \mathrm{H}\right), 1.32(\mathrm{~s}, 12 \mathrm{H})$;

${ }^{13} \mathrm{C}$ NMR $\left\{{ }^{1} \mathrm{H}\right\}\left(\mathrm{CDCl}_{3}, 75 \mathrm{MHz}\right): \delta 162.7\left(\mathrm{dd},{ }^{1} J_{\mathrm{C}-\mathrm{F}}=249.8 \mathrm{~Hz},{ }^{3} J_{\mathrm{C}-\mathrm{F}}=12.1 \mathrm{~Hz}\right), 116.8$ (m), $106.5\left(\mathrm{t},{ }^{2} J_{\mathrm{C}-\mathrm{F}}=25.2 \mathrm{~Hz}\right), 84.4,24.8 ;{ }^{11} \mathrm{~B}$ NMR $\left(\mathrm{CDCl}_{3}, 96 \mathrm{MHz}\right): \delta 31.1 ;{ }^{19} \mathrm{~F}$ NMR $\left(\mathrm{CDCl}_{3}, 282 \mathrm{MHz}\right): \delta-110.8(\mathrm{~m})$; FT-IR (neat): 2924, 2853, 1367, 1259, 1022, $800 \mathrm{~cm}^{-1}$; LRMS (\% rel. int.): m/e $240 \mathrm{M}^{+}$(16), 225 (100), 197 (13), 154 (58); Anal. Calcd for $\mathrm{C}_{12} \mathrm{H}_{15} \mathrm{BF}_{2} \mathrm{O}_{2}$ : C, 60.04; $\mathrm{H}$, 6.30. Found: $\mathrm{C}$, 59.94; $\mathrm{H}, 6.31$; HRMS Calcd for $\mathrm{C}_{12} \mathrm{H}_{15} \mathrm{BF}_{2} \mathrm{O}_{2}: 240.1133$. Found: 240.1139 .

\section{6-(4,4,5,5-tetramethyl-1,3,2-dioxaboryl)-1,3-fluorobenzene.}

$170 \mathrm{mg}$ (71\%); mp 39-40 ${ }^{\circ} \mathrm{C}$; GC-retention time 8.17 minute; ${ }^{1} \mathrm{H}$ NMR $\left(\mathrm{CDCl}_{3}\right.$, $300 \mathrm{MHz}): \delta$ 7.74-7.66 (m, 1H), 6.88-6.81 (m, 1H), 6.78-6.71 (m, 1H), $1.33(\mathrm{~s}, 12 \mathrm{H}) ;{ }^{13} \mathrm{C}$ NMR $\left\{{ }^{1} \mathrm{H}\right\}\left(\mathrm{CDCl}_{3}, 75 \mathrm{MHz}\right): \delta 168.5\left(\mathrm{dd},{ }^{1} J_{\mathrm{C}-\mathrm{F}}=175.8 \mathrm{~Hz},{ }^{3} J_{\mathrm{C}-\mathrm{F}}=12.1 \mathrm{~Hz}\right), 138.2(\mathrm{t}$, $\left.{ }^{3} J_{\mathrm{C}-\mathrm{F}}=10.1 \mathrm{~Hz}\right), 111.1\left(\mathrm{dd},{ }^{2} J_{\mathrm{C}-\mathrm{F}}=20.1 \mathrm{~Hz},{ }^{4} J_{\mathrm{C}-\mathrm{F}}=3.5 \mathrm{~Hz}\right), 103.7\left(\mathrm{dd},{ }^{2} J_{\mathrm{C}-\mathrm{F}}=27.9 \mathrm{~Hz}\right.$, $\left.{ }^{2} J_{\mathrm{C}-\mathrm{F}}=24.4 \mathrm{~Hz}\right), 84,24.8 ;{ }^{11} \mathrm{~B} \mathrm{NMR}\left(\mathrm{CDCl}_{3}, 96 \mathrm{MHz}\right): \delta 30.7 ;{ }^{19} \mathrm{~F} \mathrm{NMR}\left(\mathrm{CDCl}_{3}, 282\right.$ MHz): $\delta$-105.2 (m), -98.6 (m); FT-IR (neat): 3074, 2982, 2934, 1614, 1593, 1421, 1387, 1356, 1331, 1263, 1143, 1107, 1070, 960, 856, 733, 652, $576 \mathrm{~cm}^{-1}$; LRMS (\% rel. int.): m/e $240 \mathrm{M}^{+}$(51), 225 (100), 197 (9), 181 (45), 141 (65); Anal. Calcd for $\mathrm{C}_{12} \mathrm{H}_{15} \mathrm{BF}_{2} \mathrm{O}_{2}$ : C, 60.04; H, 6.30. Found: C, 59.71; H, 6.26; HRMS Calcd for $\mathrm{C}_{12} \mathrm{H}_{15} \mathrm{BF}_{2} \mathrm{O}_{2}: 240.1133$. Found: 240.1134 . 


\section{2-(4,4,5,5-tetramethyl-1,3,2-dioxaboryl)-1,3-fluorobenzene.}

$172 \mathrm{mg}$ (72\%); mp 50-51 ${ }^{\circ} \mathrm{C}$; GC-retention time 8.35 minute; ${ }^{1} \mathrm{H}$ NMR $\left(\mathrm{CDCl}_{3}\right.$, $300 \mathrm{MHz}): \delta$ 7.38-7.28 (m, 1H), 6.86-6.77 (m, 2H), $1.36(\mathrm{~s}, 12 \mathrm{H}) ;{ }^{13} \mathrm{C}$ NMR $\left\{{ }^{1} \mathrm{H}\right\}$ $\left(\mathrm{CDCl}_{3}, 75 \mathrm{MHz}\right): \delta 166.7\left(\mathrm{dd},{ }^{1} J_{\mathrm{C}-\mathrm{F}}=250.3 \mathrm{~Hz},{ }^{3} J_{\mathrm{C}-\mathrm{F}}=13.1 \mathrm{~Hz}\right), 132.9\left(\mathrm{t},{ }^{3} J_{\mathrm{C}-\mathrm{F}}=10.3\right.$ $\mathrm{Hz}), 111.2-110.8(\mathrm{~m}), 84.3,24.8 ;{ }^{11} \mathrm{~B}$ NMR $\left(\mathrm{CDCl}_{3}, 96 \mathrm{MHz}\right): \delta 30.6 ;{ }^{19} \mathrm{~F} \mathrm{NMR}\left(\mathrm{CDCl}_{3}\right.$, $282 \mathrm{MHz}$ ): $\delta$-100.6 (m); FT-IR (neat): 2988, 2930, 1626, 1458, 1383, 1354, 1334, 1138, 1095, 1053, 985, 850, 825, 792, 671, $559 \mathrm{~cm}^{-1}$; LRMS (\% rel. int.): m/e $240 \mathrm{M}^{+}$(26), 225 (100), 197 (5), 181 (68); Anal. Calcd for $\mathrm{C}_{12} \mathrm{H}_{15} \mathrm{BF}_{2} \mathrm{O}_{2}$ : C, 60.04; H, 6.30. Found: C, $60.02 ; \mathrm{H}, 6.42$.

\section{Borylation of 2-fluoro-4-bromobenzonitrile.}

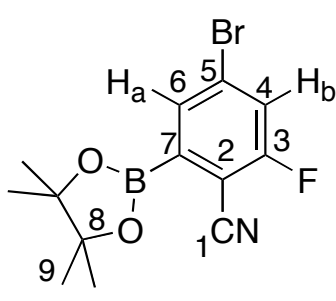

$95 \%$

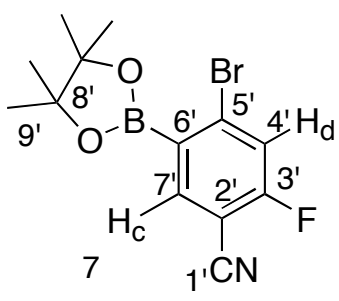

$5 \%$

General procedure B was applied to 2-fluoro-4-bromobenzonitrile (200 mg, 1 mmol, 1 equivalent) and HBPin (290 $\mu \mathrm{L}, 256 \mathrm{mg}, 2 \mathrm{mmol}, 2$ equivalent) with a reaction time of $9 \mathrm{~h}$. The ratio of the two borylated isomers in the crude reaction mixture by ${ }^{1} \mathrm{H}$ NMR spectroscopy was 95:5. A small amount of diborylated product was also observed. Solvent was removed under reduced pressure, and the crude mixture was eluted with $\mathrm{CH}_{2} \mathrm{Cl}_{2}$ through a plug of silica gel. Sublimation afforded a mixture of two isomeric borylated products $(278 \mathrm{mg}, 85 \%)$ as a white solid. The ratio of the two borylated isomers in the isolated product by by ${ }^{1} \mathrm{H}$ NMR spectroscopy was 95:5. ${ }^{1} \mathrm{H}$ NMR spectroscopy were used to assign the major isomer as 2-fluoro-4-bromo-6-(4,4,5,5tetramethyl-1,3,2-dioxaboryl)-benzonitrile. ${ }^{1} \mathrm{H}$ NMR $\left(\mathrm{CDCl}_{3}, 500 \mathrm{MHz}\right): \delta$ (major isomer) 7.77 (d, $J=1.7 \mathrm{~Hz}, 1 \mathrm{H}), 7.44\left(\mathrm{dd},{ }^{2} J_{\mathrm{H}-\mathrm{F}}=8.3 \mathrm{~Hz}, J=1.7 \mathrm{~Hz}, 1 \mathrm{H}\right), 1.35$ (br s, $12 \mathrm{H})$, (minor isomer) $7.90\left(\mathrm{~d},{ }^{3} J_{\mathrm{H}-\mathrm{F}}=7.5 \mathrm{~Hz}, 1 \mathrm{H}\right), 7.42\left(\mathrm{~d},{ }^{2} J_{\mathrm{H}-\mathrm{F}}=8.5 \mathrm{~Hz}, 1 \mathrm{H}\right), 1.35$ (br s, 
$12 \mathrm{H}) ;{ }^{13} \mathrm{C}$ NMR $\left\{{ }^{1} \mathrm{H}\right\}\left(\mathrm{CDCl}_{3}, 75 \mathrm{MHz}\right): \delta$ (major isomer) $163.3\left(\mathrm{~d},{ }^{1} J_{\mathrm{C}-\mathrm{F}}=262.8 \mathrm{~Hz}, \mathrm{C} 3\right)$, $134.6\left(\mathrm{~d},{ }^{4} J_{\mathrm{C}-\mathrm{F}}=4 \mathrm{~Hz}, \mathrm{C} 6\right), 127.8\left(\mathrm{~d},{ }^{3} J_{\mathrm{C}-\mathrm{F}}=8 \mathrm{~Hz}, \mathrm{C} 5\right), 122\left(\mathrm{~d},{ }^{2} J_{\mathrm{C}-\mathrm{F}}=23 \mathrm{~Hz}, \mathrm{C} 4\right), 112.9$ (nitrile, C1), $104.6\left(\mathrm{~d},{ }^{2} J_{\mathrm{C}-\mathrm{F}}=14.1 \mathrm{~Hz}, \mathrm{C} 2\right), 85.4,24.7 ;{ }^{11} \mathrm{~B} \mathrm{NMR}\left(\mathrm{CDCl}_{3}, 96 \mathrm{MHz}\right): \delta$ 29.94; ${ }^{19} \mathrm{~F}$ NMR $\left(\mathrm{CDCl}_{3}, 282 \mathrm{MHz}\right): \delta$ (major isomer) -103.9 (d, $J=7.9 \mathrm{~Hz}$ ); FT-IR (neat): 3086, 2986, 2934, 2237, 1590, 1557, 1462, 1408, 1375, 1358, 1329, 1140, 972, 985, 880, 843, 742, $665 \mathrm{~cm}^{-1}$; LRMS (\% rel. int.): m/e (major isomer) $325 \mathrm{M}^{+}$(15), 310 (37), 284 (67), 267 (34), 246 (31), 226 (57); Anal. Calcd for $\mathrm{C}_{13} \mathrm{H}_{14} \mathrm{BBrFNO}_{2}$ : C, 47.90; H, 4.33; N, 4.3. Found: C, 48.15, H, 4.28; N, 4.16.

\section{Borylation of 3,4-dichlorobenzonitrile.}

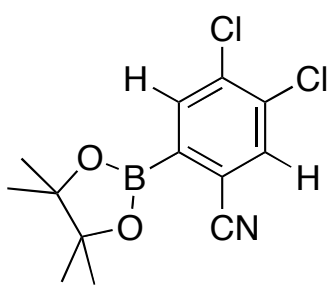

$80 \%$<smiles>CC1(C)OB(c2ccc(Cl)c(Cl)c2Cl)OC1(C)C</smiles>

$20 \%$

General procedure B was applied to 3,4-dichlorobenzonitrile (172 mg, $1 \mathrm{mmol}, 1$ equivalent) and HBPin $(218 \mu \mathrm{L}, 192 \mathrm{mg}, 1.5 \mathrm{mmol}, 1.5$ equivalent) with a reaction time of $18 \mathrm{~h}$. The ratio of the two borylated isomers in the crude reaction mixture by ${ }^{1} \mathrm{H}$ NMR spectroscopy was 80:20. Solvent was removed under reduced pressure and the crude mixture was eluted with $\mathrm{CH}_{2} \mathrm{Cl}_{2}$ through a plug of silica gel to afford a mixture of two isomeric borylated products $(265 \mathrm{mg}, 89 \%)$ as a white solid. The ratio of the two borylated isomers in the isolated product by ${ }^{1} \mathrm{H}$ NMR spectroscopy was 81:19. ${ }^{1} \mathrm{H}$ NMR spectroscopy was used to assign the major isomer as 4,5-dichloro-2-(4,4,5,5-tetramethyl1,3,2-dioxaboryl)-benzonitrile. ${ }^{1} \mathrm{H}$ NMR $\left(\mathrm{CDCl}_{3}, 500 \mathrm{MHz}\right.$ ): $\delta$ (major isomer) 7.92 (s, 1H), $7.73(\mathrm{~s}, 1 \mathrm{H}), 1.35$ (br s, 12H), (minor isomer) 7.84 (d, $J=2 \mathrm{~Hz}, 1 \mathrm{H}), 7.74$ (d, $J=2$ $\mathrm{Hz}, 1 \mathrm{H}), 1.35$ (br s, $12 \mathrm{H}) ;{ }^{13} \mathrm{C} \mathrm{NMR}\left\{{ }^{1} \mathrm{H}\right\}\left(\mathrm{CDCl}_{3}, 125 \mathrm{MHz}\right): \delta$ (major isomer) 137.7, 137.1, 135.8, 134.8, 116.9, 111.3, 85.3, 24.7, (minor isomer) 142.7, 137.6, 135, 134.3, 116.8, 111.3, 85, 24.7; ${ }^{11} \mathrm{~B}$ NMR $\left(\mathrm{CDCl}_{3}, 96 \mathrm{MHz}\right): \delta$ 29.9; FT-IR (neat): 2982, 2234, 1580, 1535, 1472, 1383, 1342, 1304, 1142, 1084, 964, 910, 850, $669 \mathrm{~cm}^{-1}$; LRMS (\% rel. int.): m/e (major isomer) $297 \mathrm{M}^{+}$(22), 282 (60), 256 (100), 239 (48), 198 (41), (minor 
isomer) $297 \mathrm{M}^{+}$(1), 282 (28), 262 (100), 220 (80); Anal. Calcd for $\mathrm{C}_{13} \mathrm{H}_{14} \mathrm{BCl}_{2} \mathrm{NO}_{2}$ : C, 52.40; H, 4.74; N, 4.70. Found: C, 52.42; H, 4.79; N, 4.55; HRMS Calcd for $\mathrm{C}_{13} \mathrm{H}_{14} \mathrm{BCl}_{2} \mathrm{NO}_{2}: 297.0495$. Found: 297.0500 .

\section{Table 3, Entry 1: Diborylation of 4-fluorobenzonitrile.}

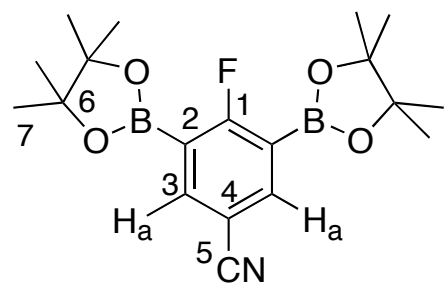

$54 \%$

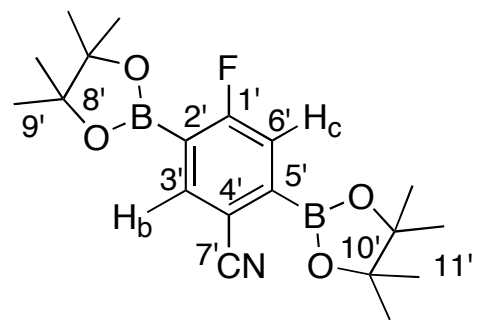

$46 \%$

General procedure $\mathrm{C}$ was applied to 4-fluorobenzonitrile (121.2 $\mathrm{mg}, 1 \mathrm{mmol}, 1$ equivalent) and HBPin (580 $\mu \mathrm{L}, 512 \mathrm{mg}, 4 \mathrm{mmol}, 4$ equivalent) with a reaction time of $24 \mathrm{~h}$. The ratio of the two isomers in the crude reaction mixture by ${ }^{1} \mathrm{H}$ NMR spectroscopy was 54:46. Solvent was removed under reduced pressure, and the crude mixture was eluted with $\mathrm{CH}_{2} \mathrm{Cl}_{2}$ through a plug of silica gel to afford a mixture of two isomeric diborylated products (343 $\mathrm{mg}, 92 \%$ ) as a white solid. The ratio of the two diborylated isomers in the isolated product by ${ }^{1} \mathrm{H}$ NMR spectroscopy was 53:47. ${ }^{1} \mathrm{H},{ }^{13} \mathrm{C}$ NMR and gHMBC spectroscopy were used to assign the major isomer as 4-fuoro-3,5-bis-(4,4,5,5tetramethyl-1,3,2-dioxaboryl)-benzonitrile. ${ }^{1} \mathrm{H}$ NMR $\left(\mathrm{CDCl}_{3}, 500 \mathrm{MHz}\right): \delta$ (major isomer) $8.1\left(\mathrm{~d},{ }^{4} J_{\mathrm{H}-\mathrm{F}}=4.9 \mathrm{~Hz}, 2 \mathrm{H}, \mathrm{H}_{\mathrm{a}}\right), 1.28(\mathrm{br} \mathrm{s}, 24 \mathrm{H})$, (minor isomer) $8.04\left(\mathrm{~d},{ }^{4} J_{\mathrm{H}-\mathrm{F}}=\right.$ $\left.5.4 \mathrm{~Hz}, 1 \mathrm{H}, \mathrm{H}_{\mathrm{b}}\right), 7.46\left(\mathrm{~d},{ }^{3} J_{\mathrm{H}-\mathrm{F}}=8.8 \mathrm{~Hz}, 1 \mathrm{H}, \mathrm{H}_{\mathrm{c}}\right), 1.32(\mathrm{~s}, 12 \mathrm{H}), 1.31(\mathrm{~s}, 12 \mathrm{H}) ;{ }^{13} \mathrm{C} \mathrm{NMR}$ $\left\{{ }^{1} \mathrm{H}\right\}\left(\mathrm{CDCl}_{3}, 125 \mathrm{MHz}\right): \delta$ (major isomer) $172\left(\mathrm{~d},{ }^{1} J_{\mathrm{C}-\mathrm{F}}=266 \mathrm{~Hz}, \mathrm{C} 1\right), 143.9\left(\mathrm{~d},{ }^{3} J_{\mathrm{C}-\mathrm{F}}=\right.$ $10.9 \mathrm{~Hz}, \mathrm{C} 3$ ), 117.9 (nitrile C5), 108 (d, ${ }^{4} J_{\mathrm{C}-\mathrm{F}}=3.6 \mathrm{~Hz}, \mathrm{C} 4$ ), 84.4 (C6), 24.7(C7), (minor isomer) $168.1\left(\mathrm{~d},{ }^{1} J_{\mathrm{C}-\mathrm{F}}=261.4 \mathrm{~Hz}, \mathrm{C} 1^{\prime}\right), 142.6\left(\mathrm{~d},{ }^{3} J_{\mathrm{C}-\mathrm{F}}=8.8 \mathrm{~Hz}, \mathrm{C} 3^{\prime}\right), 122.6\left(\mathrm{~d},{ }^{2} J_{\mathrm{C}-\mathrm{F}}=\right.$ $23.8 \mathrm{~Hz}, \mathrm{C}^{\prime}$ ), 118.1 (nitrile $\mathrm{C}^{\prime}$ ), $112.6\left(\mathrm{~d},{ }^{4} J_{\mathrm{C}-\mathrm{F}}=3.6 \mathrm{~Hz}, \mathrm{C} 4^{\prime}\right.$ ), 85, 84.5, $24.7 ;{ }^{11} \mathrm{~B}$ $\mathrm{NMR}\left(\mathrm{CDCl}_{3}, 96 \mathrm{MHz}\right): \delta 29.8 ;{ }^{19} \mathrm{~F} \mathrm{NMR}\left(\mathrm{CDCl}_{3}, 282 \mathrm{MHz}\right): \delta$ (major isomer) -81.8 (m), (minor isomer) -94.9 (m);FT-IR (neat): 2980, 2934, 2232, 1599, 1497, 1437, 1414, 1373, 1334, 1267, 1215, 1142, 1095, 966, 889, 848, $584 \mathrm{~cm}^{-1}$; LRMS (\% rel. int.): m/e (major isomer) $373 \mathrm{M}^{+}$(4), 358 (59), 353 (100), 315 (22), 253 (45), (minor isomer) 373 
$\mathrm{M}^{+}$(32), 358 (68), 331 (100), 315 (53), 274 (39); Anal. Calcd for $\mathrm{C}_{19} \mathrm{H}_{26} \mathrm{~B}_{2} \mathrm{FNO}_{4}$ : C, 61.17; H, 7.03; N, 3.75. Found: C, 61.37,; H, 6.83; N, 3.82.

Table 3, Entry 2: Diborylation of 4-methoxybenzonitrile.
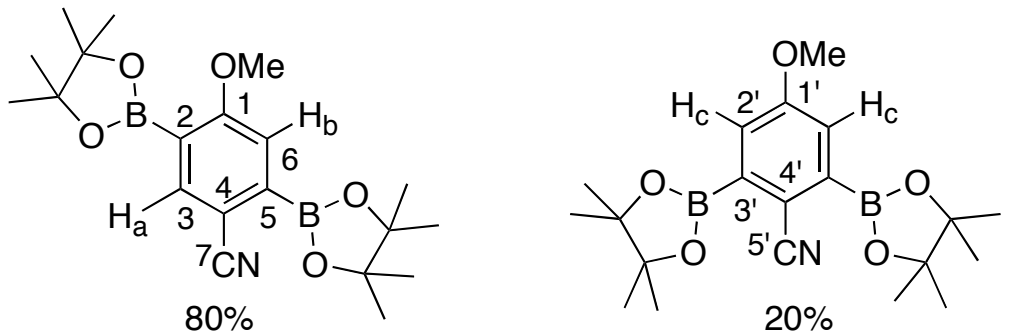

General procedure $\mathrm{C}$ was applied to 4-methoxybenzonitrile (133.2 $\mathrm{mg}, 1 \mathrm{mmol}, 1$ equivalent) and HBPin $\left(580 \mu \mathrm{L}, 512 \mathrm{mg}, 4 \mathrm{mmol}, 4\right.$ equivalent) at $60{ }^{\circ} \mathrm{C}$ for $48 \mathrm{~h}$. The ratio of the two diborylated isomers in the crude reaction mixture by ${ }^{1} \mathrm{H}$ NMR spectroscopy was 80:20. Solvent was removed under reduced pressure, and the crude mixture was eluted with $\mathrm{CH}_{2} \mathrm{Cl}_{2}$ through a plug of silica gel to afford a mixture of two isomeric diborylated products $(310 \mathrm{mg}, 81 \%)$ as a white solid. The ratio of the two diborylated isomers in the isolated product by ${ }^{1} \mathrm{H}$ NMR spectroscopy was 80:20. ${ }^{1} \mathrm{H}$ NMR and gHMBC spectroscopy were used to assign the major isomer as 4-methoxy-2,5bis-(4,4,5,5-tetramethyl-1,3,2-dioxaboryl)-benzonitrile. ${ }^{1} \mathrm{H}$ NMR $\left(\mathrm{C}_{6} \mathrm{D}_{6}, 500 \mathrm{MHz}\right): \delta$ (major isomer) $8.42\left(\mathrm{~s}, 1 \mathrm{H}, \mathrm{H}_{\mathrm{a}}\right), 7.36\left(\mathrm{~s}, 1 \mathrm{H}, \mathrm{H}_{\mathrm{b}}\right), 3.20(\mathrm{~s}, 3 \mathrm{H}), 1.10(\mathrm{br} \mathrm{s}, 12 \mathrm{H}), 1.06(\mathrm{br}$ $\mathrm{s}, 12 \mathrm{H})$, (minor isomer) $7.61\left(\mathrm{~s}, 2 \mathrm{H}, \mathrm{H}_{\mathrm{c}}\right), 3.08(\mathrm{~s}, 3 \mathrm{H}), 1.11(\mathrm{br} \mathrm{s}, 12 \mathrm{H}) ;{ }^{13} \mathrm{C} \mathrm{NMR}\left\{{ }^{1} \mathrm{H}\right\}$ $\left(\mathrm{C}_{6} \mathrm{D}_{6}, 125 \mathrm{MHz}\right): \delta$ (major isomer) $166.3(\mathrm{C} 1), 143.1$ (C3), 137.4 (br, C5), 122.1 (br, C2), 119.32 (nitrile, C7), 117.36 (C6), 110.1 (C4), 84.8, 83.8, 24.79, 24.76, (minor isomer) $160.8\left(\mathrm{Cl}^{\prime}\right), 123.4\left(\mathrm{C}^{\prime}\right), 118.6$ (nitrile $\left.\mathrm{C}^{\prime}\right), 114.9\left(\mathrm{C} 4^{\prime}\right), 84.624 .8 ;{ }^{11} \mathrm{~B}$ NMR $\left(\mathrm{CDCl}_{3}, 96 \mathrm{MHz}\right): \delta$ 30.90; FT-IR (neat): 2980, 2936, 2224, 1601, 1503, 1398, 1373, 1337, 1244, 1142, 1094, 964, $858 \mathrm{~cm}^{-1}$; LRMS (\% rel. int.): $\mathrm{m} / \mathrm{e} 385 \mathrm{M}^{+}$(100), 370 (63), 343 (98), 327 (53), 286 (41); Anal. Calcd for $\mathrm{C}_{20} \mathrm{H}_{29} \mathrm{~B}_{2} \mathrm{NO}_{5}$ : C, 62.38; H, 7.59; N, 3.64. Found: C, 62.36; H, 7.41; N, 3.69 . 
Table 3, Entry 3: Diborylation of 4-chlorobenzonitrile.

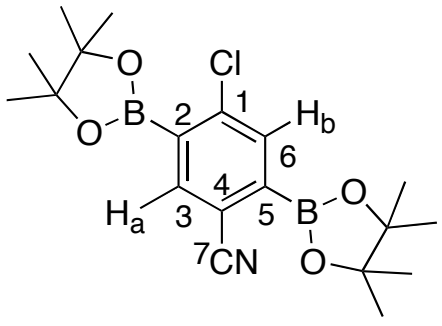

$80 \%$

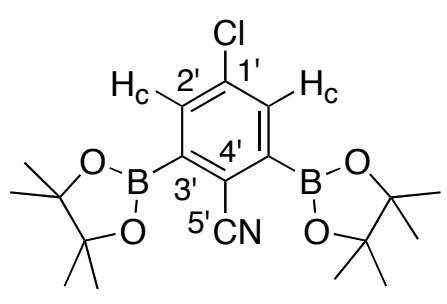

$20 \%$

General procedure $\mathrm{C}$ was applied to 4-chlorobenzonitrile (137.6 mg, $1 \mathrm{mmol}, 1$ equivalent) and HBPin (580 $\mu \mathrm{L}, 512 \mathrm{mg}, 4 \mathrm{mmol}, 4$ equivalent) with a reaction time of $48 \mathrm{~h}$. The ratio of the two diborylated isomers in the crude reaction mixture by GC-FID was 80:20. Solvent was removed under reduced pressure, and the crude mixture was eluted with $\mathrm{CH}_{2} \mathrm{Cl}_{2}$ through a plug of silica gel to afford a mixture of two isomeric diborylated products $(320 \mathrm{mg}, 82 \%)$ as a white solid. The ratio of the two diborylated isomers in the isolated product by GC-FID was 80:20. ${ }^{1} \mathrm{H}$ NMR and gHMBC spectroscopy were used to assign the major isomer as 4-chloro-2,5-bis-(4,4,5,5tetramethyl-1,3,2-dioxaboryl)-benzonitrile. ${ }^{1} \mathrm{H} \mathrm{NMR}\left(\mathrm{CDCl}_{3}, 500 \mathrm{MHz}\right): \delta$ (major isomer) $7.97\left(\mathrm{~s}, 1 \mathrm{H}, \mathrm{H}_{\mathrm{a}}\right), 7.80\left(\mathrm{~s}, 1 \mathrm{H}, \mathrm{H}_{\mathrm{b}}\right), 1.35$ (br s, 12H), 1.344 (br s, 12H), (minor isomer) $7.83\left(\mathrm{~s}, 2 \mathrm{H}, \mathrm{H}_{\mathrm{c}}\right), 1.349$ (br s, $\left.12 \mathrm{H}\right) ;{ }^{13} \mathrm{C} \mathrm{NMR}\left\{{ }^{1} \mathrm{H}\right\}\left(\mathrm{CDCl}_{3}, 125 \mathrm{MHz}\right): \delta$ (major isomer) 143.6 (C1), 141.2 (C3), 136.5 (C6), 118.2 (nitrile, C7), 114.8 (C4), 85.1, 84.8, 24.7, (minor isomer) 137.7 (C1' ), 137.3 (C2' ), 119.7(C4' ), 117.6 (nitrile $\mathrm{C}^{\prime}$ ), 85.04 $24.7 ;{ }^{11} \mathrm{~B}$ NMR $\left(\mathrm{CDCl}_{3}, 96 \mathrm{MHz}\right): \delta$ 30.25; FT-IR (neat): 2980, 2230, 1591, 1383, 1341, 1269, 1142, 1122, 1088, 962, $855 \mathrm{~cm}^{-1}$; LRMS (\% rel. int.): m/e (major isomer) $389 \mathrm{M}^{+}$ (30), 374 (57), 354 (100), 347 (88), 331 (39), 312 (37), 290 (35), (minor isomer) $389 \mathrm{M}^{+}$ (36), 374 (64), 348 (23), 290 (53), 248 (49), 207 (100); Anal. Calcd for $\mathrm{C}_{19} \mathrm{H}_{26} \mathrm{~B}_{2} \mathrm{ClNO}_{4}$ : C, 58.59; H, 6.73; N, 3.6. Found: C, 58.27, H, 6.78; N, 3.41; HRMS Calcd for $\mathrm{C}_{19} \mathrm{H}_{26} \mathrm{~B}_{2} \mathrm{ClNO}_{4}$ : 389.1736. Found: 389.1746. 
Table 3, Entry 4: Diborylation of 1,4-dicyanobenzene.

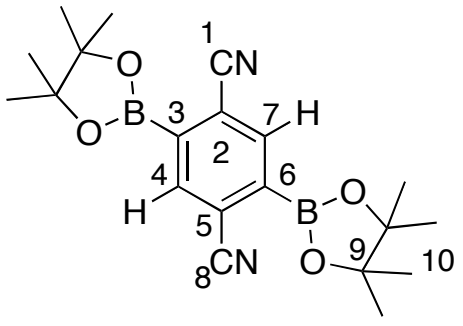

$93 \%$

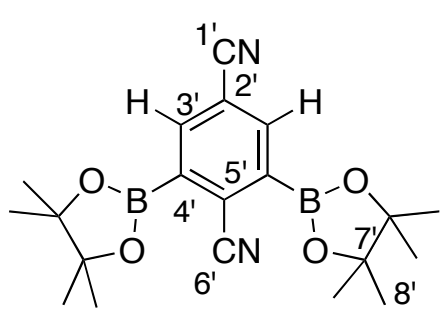

$7 \%$

General procedure $\mathrm{C}$ was applied to 1,4-dicyanobenzene (128.2 $\mathrm{mg}, 1 \mathrm{mmol}, 1$ equivalent) and HBPin (580 $\mu \mathrm{L}, 512 \mathrm{mg}, 4 \mathrm{mmol}, 4$ equivalent) with a reaction time of $20 \mathrm{~h}$. The ratio of the two diborylated isomers in the crude reaction mixture by GC-FID was 93:7. Solvent was removed under reduced pressure. Crystallization from $\mathrm{THF} /$ pentane gave the major isomer $\left(270 \mathrm{mg}, 71 \%\right.$ ) as a white solid, $\mathrm{mp} 242-245{ }^{\circ} \mathrm{C} .{ }^{13} \mathrm{C}$ NMR spectroscopy were used to assign the major isomer as 2,5-bis-(4,4,5,5-tetramethyl1,3,2-dioxaboryl)-1,4-dicyanobenzene. ${ }^{1} \mathrm{H} \mathrm{NMR}\left(\mathrm{CDCl}_{3}, 500 \mathrm{MHz}\right): \delta$ (major isomer) $8.16(\mathrm{~s}, 2 \mathrm{H}), 1.37$ (br s, 24H), (minor isomer) 8.15 (s, 2H,), 1.37 (br s, 24H); ${ }^{13} \mathrm{C} \mathrm{NMR}$ $\left\{{ }^{1} \mathrm{H}\right\}\left(\mathrm{CDCl}_{3}, 75 \mathrm{MHz}\right): \delta$ (major isomer) 140.1 (C4, 2C), 135.4 (br, C3, 2C), 120.1 (C2, 2C), 117.4 (nitrile, $\mathrm{C} 1,2 \mathrm{C}), 85.4,24.7 ;{ }^{11} \mathrm{~B} \mathrm{NMR}\left(\mathrm{CDCl}_{3}, 96 \mathrm{MHz}\right): \delta 30.16$; FT-IR (neat): 2984, 2230, 1497, 1416, 1391, 1347, 1287, 1267, 1169, 1140, 1100, 962, 927, $855,814,711,598 \mathrm{~cm}^{-1}$; LRMS (\% rel. int.): m/e (major isomer) $380 \mathrm{M}^{+}$(35), 365 (69), 339 (100), 322 (69), 281 (46); Anal. Calcd for $\mathrm{C}_{20} \mathrm{H}_{26} \mathrm{~B}_{2} \mathrm{~N}_{2} \mathrm{O}_{4}$ : C, 63.21; H, 6.90; N, 7.37. Found: C, 63.39, H, 7.19; N, 7.02.

Table 3, Entry 5: Diborylation of 4-(trifluoromethyl)-benzonitrile.

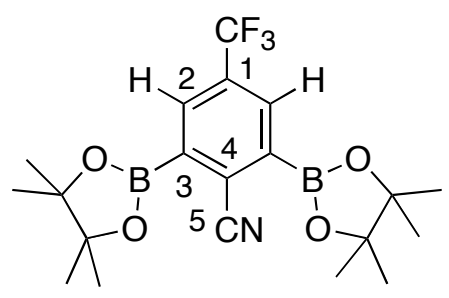


General procedure C was applied to 4-(trifluoromethyl)-benzonitrile (171.2 mg, 1 mmol, 1 equivalent) and HBPin $(580 \mu \mathrm{L}, 512 \mathrm{mg}, 4 \mathrm{mmol}, 4$ equivalent) with a reaction time of $36 \mathrm{~h}$. Solvent was removed under reduced pressure, and the crude mixture was eluted with $\mathrm{CH}_{2} \mathrm{Cl}_{2}$ through a plug of silica gel to afford single diborylated isomer (350 $\mathrm{mg}, 83 \%$ ) as a white solid. ${ }^{13} \mathrm{C}$ NMR spectroscopy were used to assign the single isomer as 4-(trifluoromethyl)-2,6-bis-(4,4,5,5-tetramethyl-1,3,2-dioxaboryl)-benzonitrile. ${ }^{1} \mathrm{H}$ $\mathrm{NMR}\left(\mathrm{CDCl}_{3}, 300 \mathrm{MHz}\right): \delta 8.12(\mathrm{~s}, 2 \mathrm{H}), 1.37$ (br s, $\left.12 \mathrm{H}\right) ;{ }^{13} \mathrm{C} \mathrm{NMR}\left\{{ }^{1} \mathrm{H}\right\}\left(\mathrm{CDCl}_{3}, 75\right.$ MHz): $\delta 133.9\left(\mathrm{q},{ }^{3} J_{\mathrm{C}-\mathrm{F}}=3 \mathrm{~Hz}, \mathrm{C} 2\right), 132\left(\mathrm{q},{ }^{2} J_{\mathrm{C}-\mathrm{F}}=32.2 \mathrm{~Hz}, \mathrm{C} 1\right), 125$ (nitrile, C5), 123.3 $\left(\mathrm{q},{ }^{1} J_{\mathrm{C}-\mathrm{F}}=273 \mathrm{~Hz}, \mathrm{CF}_{3}\right), 117.1(\mathrm{C} 4), 85.2,24.7 ;{ }^{11} \mathrm{~B} \mathrm{NMR}\left(\mathrm{CDCl}_{3}, 96 \mathrm{MHz}\right): \delta 30.14$;

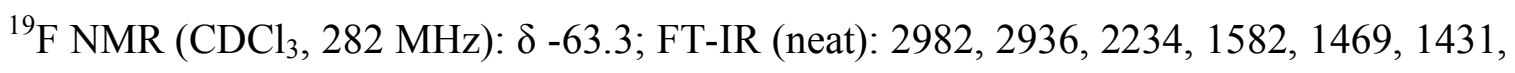
$1383,1373,1334,1319,1290,1269,1245,1140,1080,966,883,846,690 \mathrm{~cm}^{-1}$; LRMS (\% rel. int.): m/e $423 \mathrm{M}^{+}$(45), 408 (100), 382 (36), 324 (45), 282 (62); Anal. Calcd for $\mathrm{C}_{20} \mathrm{H}_{26} \mathrm{~B}_{2} \mathrm{~F}_{3} \mathrm{NO}_{4}$ : C, 56.78; H, 6.19; N, 3.31. Found: C, 56.81; H, 5.92; N, 3.29; HRMS Calcd for $\mathrm{C}_{20} \mathrm{H}_{26} \mathrm{~B}_{2} \mathrm{~F}_{3} \mathrm{NO}_{4}$ : 423.2000. Found: 423.1999 .

\section{Borylation of PhBPin.}

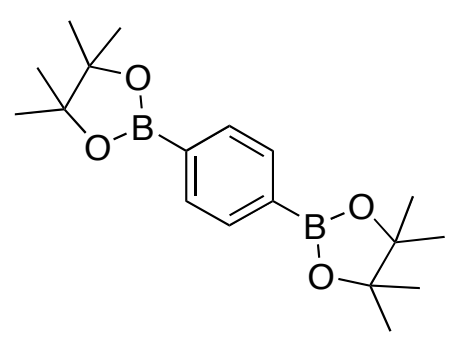

$64.6 \%$

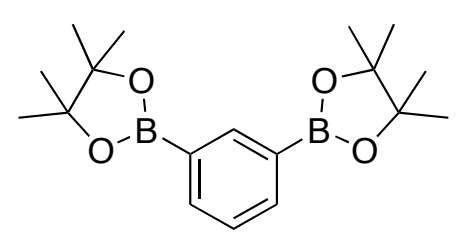

$35.4 \%$

General procedure B was applied to benzene (78.1 $\mathrm{mg}, 1 \mathrm{mmol}, 1$ equivalent) and HBPin (175 $\mu \mathrm{L}, 153.6 \mathrm{mg}, 1.2 \mathrm{mmol}, 1.2$ equivalents) with a reaction time of $24 \mathrm{~h}$. The first equivalent of borane generates PhBpin in situ and the remaining 0.2 equivalents give the two diborylated isomers. PhBpin and the two diborylated isomers were present at the end of reaction. The GC-FID ratio of the two diborylated isomers was 64.6:35.4. A mixture of the two diborylated isomers was isolated $(51 \mathrm{mg}) .{ }^{1} \mathrm{H}$ NMR spectroscopy was used to assign the major isomer as 1,4-bis-(4,4,5,5-tetramethyl-1,3,2-dioxaboryl)benzene. ${ }^{1} \mathrm{H}$ NMR $\left(\mathrm{CDCl}_{3}, 300 \mathrm{MHz}\right): \delta$ (major/para isomer) $7.78(\mathrm{~s}, 4 \mathrm{H}), 1.32(\mathrm{~s}, 12 \mathrm{H})$, 
(minor/meta isomer) $8.25(\mathrm{~d}, J=1 \mathrm{~Hz}, 1 \mathrm{H}), 7.88(\mathrm{dd}, J=7.3,1.5 \mathrm{~Hz}, 2 \mathrm{H}), 7.35(\mathrm{td}, J=$ $7.3,1 \mathrm{~Hz}, 1 \mathrm{H}), 1.31(\mathrm{~s}, 12 \mathrm{H})$. The spectra were in agreement with those described in the literature.

\section{Borylation of 1,5-dimethyl-2-pyrrolecarbonitrile.}

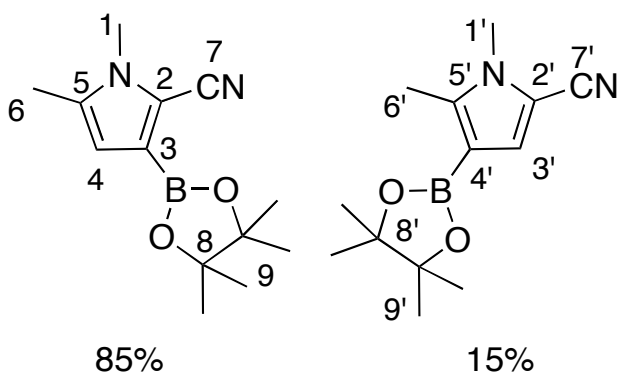

General procedure B was applied to 1,5-dimethyl-2-pyrrolecarbonitrile (240.3 mg, 2 mmol, 1 equivalent) and HBPin (435 $\mu \mathrm{L}, 384 \mathrm{mg}, 3 \mathrm{mmol}, 1.5$ equivalents) with a reaction time of $16 \mathrm{~h}$. The ratio of the two isomers in the crude reaction mixture by GC was 85:15. Solvent was removed under reduced pressure, and the crude mixture was eluted with $\mathrm{CH}_{2} \mathrm{Cl}_{2}$ through a plug of silica gel to afford a mixture of two isomeric borylated products $(394 \mathrm{mg}, 80 \%)$ as a white solid. The ratio of the two isomers in the isolated product by GC was 82:18. The NOESY1D and gHMBC spectra (Figure 9) were used to assign the major isomer as 1,5-dimethyl-3-(4,4,5,5-tetramethyl-1,3,2-dioxaboryl)2-pyrrolecarbonitrile. ${ }^{1} \mathrm{H} \mathrm{NMR}\left(\mathrm{CDCl}_{3}, 300 \mathrm{MHz}\right): \delta$ (major isomer) $6.21\left(\mathrm{~d},{ }^{4} \mathrm{~J}=0.7 \mathrm{~Hz}\right.$, $1 \mathrm{H}$, pyrrol ring $\mathrm{H}), 3.63$ (s, $\left.3 \mathrm{H}, \mathrm{N}-\mathrm{CH}_{3}\right), 2.21$ (d, ${ }^{4} \mathrm{~J}=0.7 \mathrm{~Hz}, 3 \mathrm{H}, \mathrm{CH}_{3}$ on pyrrol ring), 1.30 (br s, 12H), (minor isomer) $7.03(\mathrm{~s}, 1 \mathrm{H}$, pyrrol ring $\mathrm{H}), 3.6\left(\mathrm{~s}, 3 \mathrm{H}, \mathrm{N}-\mathrm{CH}_{3}\right), 2.41(\mathrm{~s}$, $3 \mathrm{H}, \mathrm{CH}_{3}$ on pyrrol ring), $1.27(\mathrm{~s}, 12 \mathrm{H}) ;{ }^{13} \mathrm{C} \mathrm{NMR}\left\{{ }^{1} \mathrm{H}\right\}\left(\mathrm{CDCl}_{3}, 125 \mathrm{MHz}\right): \delta$ (major isomer) 135.4 (C5), 114 (C4), 113.8 (nitrile C7), 109.6 (C2), 83.0 (C8), 32.0 (C1), 24.3 (C9), 11.8 (C6), (minor isomer) 143.9 (C5' ), 125.2 (C3' ), 113.7 (nitrile C7' ), 103.8 (C2' ), 82.6 (C8' ), 31.8 (C1' ), $24.4\left(\mathrm{C}^{\prime}\right), 11.9$ (C6' ); ${ }^{11} \mathrm{~B}$ NMR ( $\left.\mathrm{CDCl}_{3}, 96 \mathrm{MHz}\right): \delta$ 32.75; FT-IR (neat): 2980. 2934, 2735, 1561, 1501, 1441, 1408, 1390, 1379, 1371, 1313, 1262, 1187, 1167, 1144, 1111, 1017, 860, 835, $708 \mathrm{~cm}^{-1}$; LRMS (\% rel. int.): m/e (major isomer) $246 \mathrm{M}^{+}$(100), 231 (19), 203 (12), 189 (16), 160 (13), 146 (20), (minor isomer) $246 \mathrm{M}^{+}$(100), 231 (20), 189 (51), 160 (21), 146 (43); Anal. Calcd for $\mathrm{C}_{13} \mathrm{H}_{19} \mathrm{BN}_{2} \mathrm{O}_{2}$ : C, 63.44; H, 7.78; N, 11.38. Found: C, 63.34; H, 7.78; N, 11.34 . 


\section{Borylation of 5-methyl-2-furonitrile.}

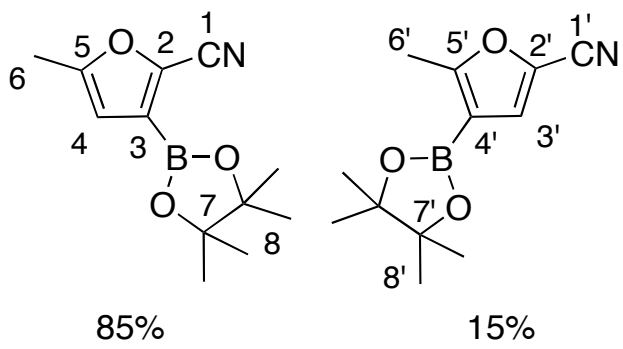

General procedure B was applied to 5-methyl-2-furonitrile (210 uL, $214.2 \mathrm{mg}, 2$ mmol, 1 equivalent) and HBPin (435 $\mu \mathrm{L}, 384 \mathrm{mg}, 3 \mathrm{mmol}, 1.5$ equivalents) with a reaction time of $0.5 \mathrm{~h}$. The ratio of the two isomers in the crude reaction mixture by GC was $85: 15$. Solvent was removed under reduced pressure, and the crude mixture was eluted with $\mathrm{CH}_{2} \mathrm{Cl}_{2}$ through a plug of silica gel to afford a mixture of two isomeric borylated products $(456 \mathrm{mg}, 97 \%$ ) as a white solid. The ratio of the two isomers in the isolated product by GC was 90:10. The NOESY1D and gHMBC spectra (Figure 10) were used to assign the major isomer as 5-methyl-3-(4,4,5,5-tetramethyl-1,3,2-dioxaboryl)-2furonitrile. ${ }^{1} \mathrm{H} \mathrm{NMR}\left(\mathrm{CDCl}_{3}, 300 \mathrm{MHz}\right): \delta$ (major isomer) $6.23\left(\mathrm{~d},{ }^{4} \mathrm{~J}=1.0 \mathrm{~Hz}, 1 \mathrm{H}\right.$, furan ring $\mathrm{H}$ ), $2.29\left(\mathrm{~d},{ }^{4} \mathrm{~J}=1.0 \mathrm{~Hz}, 3 \mathrm{H}, \mathrm{CH}_{3}\right.$ on furan ring), 1.27 (br s, 12H), (minor isomer) $7.12(\mathrm{~s}, 1 \mathrm{H}$, furan ring $\mathrm{H}), 2.46\left(\mathrm{~s}, 3 \mathrm{H}, \mathrm{CH}_{3}\right.$ on furan ring), $1.25(\mathrm{~s}, 12 \mathrm{H}) ;{ }^{13} \mathrm{C} \mathrm{NMR}\left\{{ }^{1} \mathrm{H}\right\}$ $\left(\mathrm{CDCl}_{3}, 75 \mathrm{MHz}\right): \delta$ (major isomer) 157.6 (C5), 130.4 (C2), 111.9 (nitrile $\left.\mathrm{C} 1\right), 111.5$ (C4), 84.3 (C7), 24.6 (C8), 13.4 (C6), (minor isomer) 167 (C5' ), 127.4 (C3' ), 124.1 (C2' ), 111.7 (nitrile $\mathrm{C}^{\prime}$ ), 83.8 (C7'), 24.7 (C8' ), 14.2 (C6' ); ${ }^{11} \mathrm{~B}$ NMR $\left(\mathrm{CDCl}_{3}, 96\right.$ $\mathrm{MHz}): \delta$ 28.9; FT-IR (neat): 2982, 2934, 1595, 1543, 1446, 1428, 1408, 1392, 1381, 1373, 1335, 1302, 1228, 1169, 1143, 1043, 963, 855, 804, $712 \mathrm{~cm}^{-1}$; LRMS (\% rel. int.): m/e (major isomer) $233 \mathrm{M}^{+}$(89), 218 (42), 203 (20), 190 (64), 175 (59), 149 (100), 134 (47), (minor isomer) $233 \mathrm{M}^{+}$(100), 218 (81), 191 (54), 175 (62), 149 (53), 133 (63); Anal. Calcd for $\mathrm{C}_{12} \mathrm{H}_{16} \mathrm{BNO}_{3}$ : C, 61.84; H, 6.92; N, 6.01. Found: C, 62.25; H, 7.0; N, 5.80 . 


\section{Borylation of 5-bromo-2-cyanopyridine.}

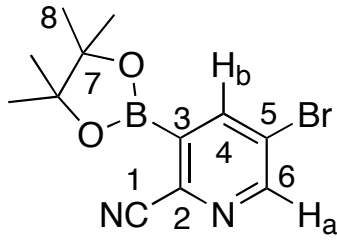

$67 \%$

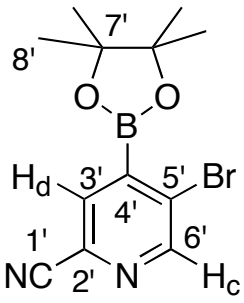

$33 \%$

General procedure B was applied to 5-bromo-2-cyanopyridine (183 mg, $1 \mathrm{mmol}$, 1 equivalent) and HBPin ( $290 \mu \mathrm{L}, 256 \mathrm{mg}, 2 \mathrm{mmol}, 2$ equivalent) with a reaction time of 18h. The ratio of the two isomers in the crude reaction mixture by ${ }^{1} \mathrm{H}$ NMR spectroscopy was 67:33. Kugelrohr distillation furnished a mixture of the two isomeric borylated products $(253.5 \mathrm{mg}, 81 \%)$ as a white solid. The ratio of the two isomers in the isolated product by ${ }^{1} \mathrm{H}$ NMR spectroscopy was $64: 36 .{ }^{1} \mathrm{H}$ NMR and gHMBC spectroscopy were used to assign the major isomer as 5-bromo-3-(4,4,5,5-tetramethyl-1,3,2-dioxaboryl)-2cyanopyridine. ${ }^{1} \mathrm{H} \mathrm{NMR}\left(\mathrm{CDCl}_{3}, 300 \mathrm{MHz}\right): \delta$ (major isomer) $8.76(\mathrm{~d}, J=2.4 \mathrm{~Hz}, 1 \mathrm{H}$, $\left.\mathrm{H}_{\mathrm{a}}\right), 8.28\left(\mathrm{~d}, J=2.4 \mathrm{~Hz}, 1 \mathrm{H}, \mathrm{H}_{\mathrm{b}}\right), 1.37$ (br s, 12H), (minor isomer) $8.76\left(\mathrm{~s}, 1 \mathrm{H}, \mathrm{H}_{\mathrm{c}}\right), 7.85$ $\left(\mathrm{s}, 1 \mathrm{H}, \mathrm{H}_{\mathrm{d}}\right), 1.36(\mathrm{~s}, 12 \mathrm{H}) ;{ }^{13} \mathrm{C} \mathrm{NMR}\left\{{ }^{1} \mathrm{H}\right\}\left(\mathrm{CDCl}_{3}, 75 \mathrm{MHz}\right): \delta$ (major isomer) 153.6 (C6), 145.8 (C4), 136.1 (C2), 124.4 (C5), 116.6 (nitrile C1), 85.7 (C7), 24.8 (C8), (minor isomer) 153.1 (C6' ), 134.5 (C3' ), 131.4 (C2' ), 130.1 (C5' ), 116.7 (nitrile $\mathrm{C}^{\prime}$ ) $), 85.6$ (C7' ), 24.8 (C8' ); ${ }^{11} \mathrm{~B}$ NMR $\left(\mathrm{CDCl}_{3}, 96 \mathrm{MHz}\right): \delta$ 29.5; FT-IR (neat): 3048, 2979, 2244, 1566, 1539, 1416, 1383, 1342, 1318, 1269, 1142, 1069, 1026, 964, 872, 847, $771 \mathrm{~cm}^{-1}$; LRMS (\% rel. int.): m/e (major isomer) $308 \mathrm{M}^{+}$(41), $310\left(\mathrm{M}^{2+} 37\right), 293$ (95), 267 (96), 250 (65), 229 (34), 209 (42), (minor isomer) $308 \mathrm{M}^{+}$(7), 293 (33), 267 (17), 229 (100), 187 (91); Anal. Calcd for $\mathrm{C}_{12} \mathrm{H}_{14} \mathrm{BBrN}_{2} \mathrm{O}_{2}$ : C, 46.65; H, 4.57; N, 9.07. Found: C, 46.52; H, 4.48; N, 8.761. 


\section{References:}

1. Knochel, P.; Davidson, J.; Tucker, C. E. J. Org. Chem. 1992, 57, 3482-3485.

2. Uson, R.; Oro, L. A. Inorg. Synth. 1985, 23, 126-130.

3. Shaka, A. J.; Keeler, J.; Hwang, T. L.; Stonehouse, J.; Stott, K. J. Am. Chem. Soc. 1995, 117, 4199-4200.

4. Miyaura, N.; Ishida, K.; Ishiyama, T. Tetrahedron 2001, 57, 9813-9816.

5. Maleczka, R. E., Jr; Shi, F.; Holmes, D. Smith, M. R., III. J. Am. Chem. Soc. 2003, $125,7792-7793$.

6. Seki, T. et al. Chem. Phar. Bull. 1998, 46(1), 84-96. 


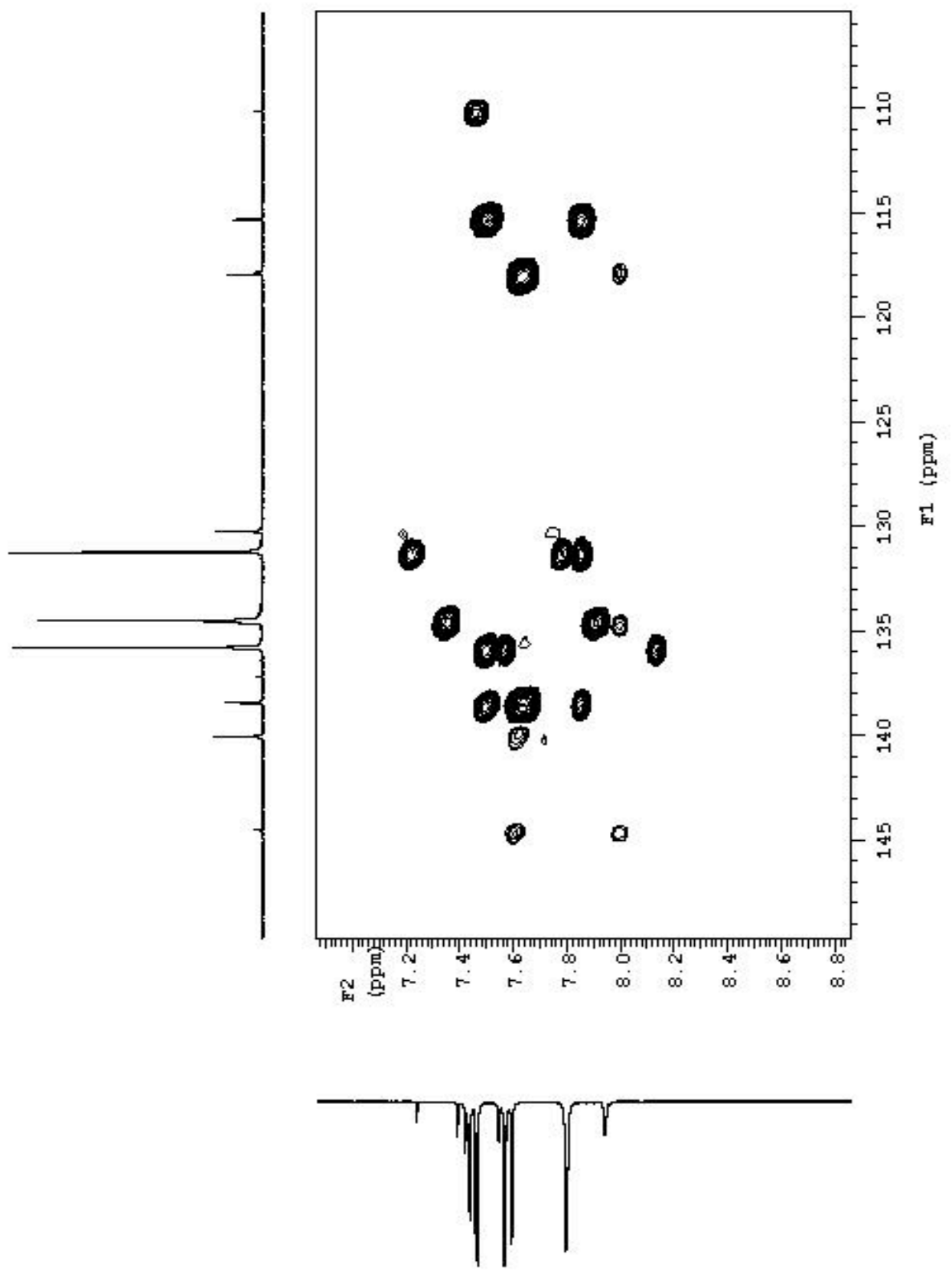

Figure 1. gHMBC spectrum of the borylation products of 4-chlorobenzonitrile. 


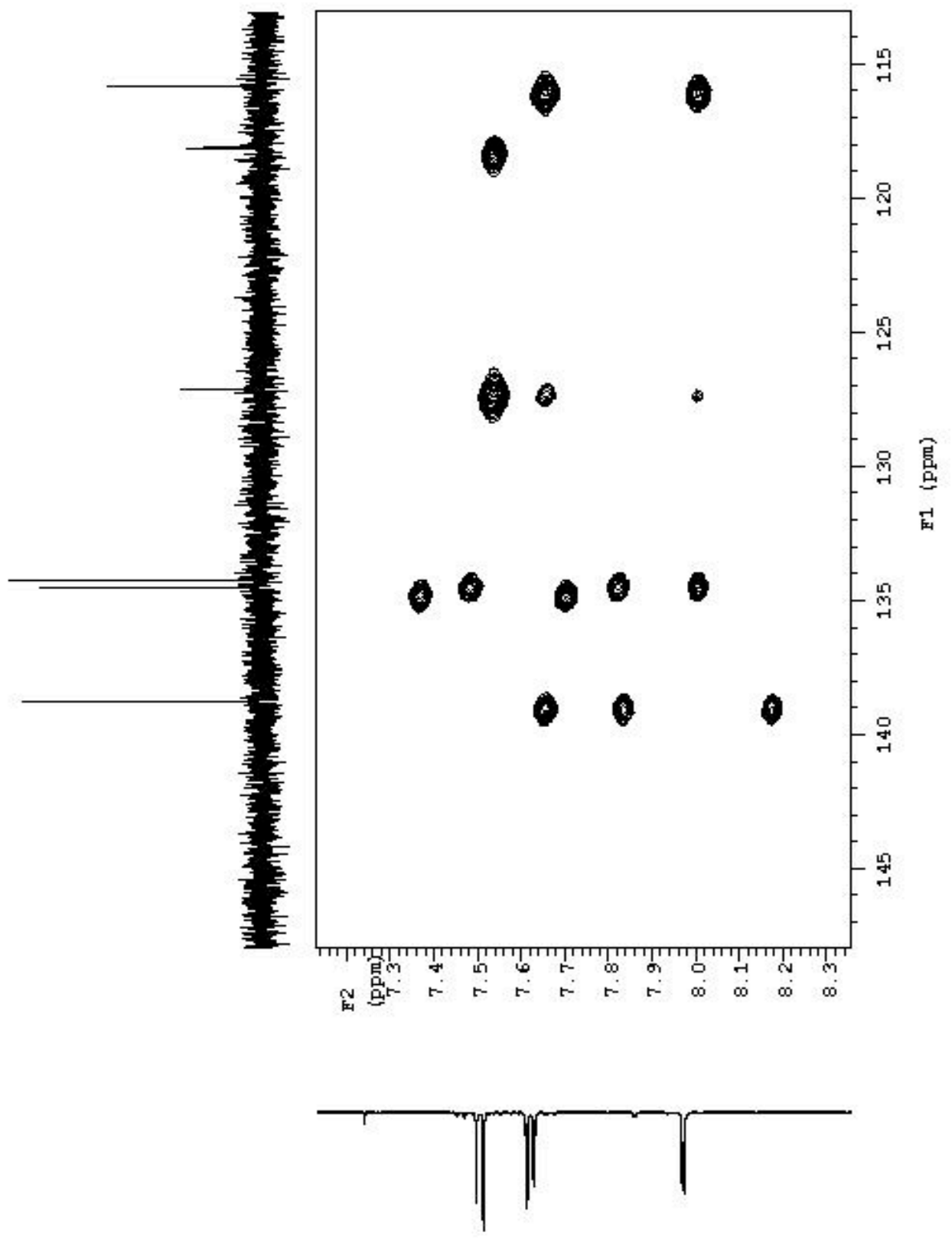

Figure 2. gHMBC spectrum of the borylation products of 4-bromobenzonitrile. 


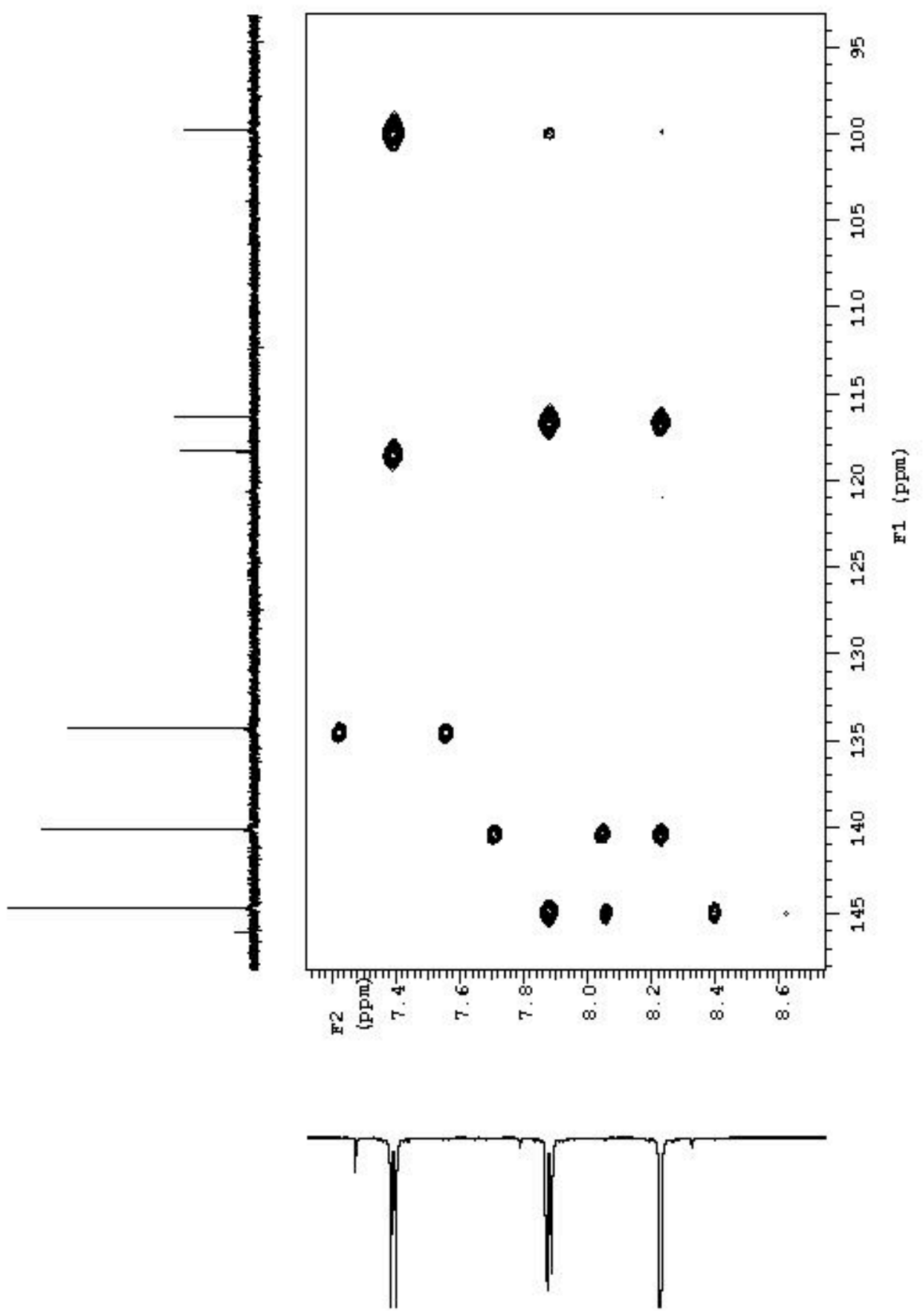

Figure 3. gHMBC spectrum of the borylation product of 4-Iodobenzonitrile. 

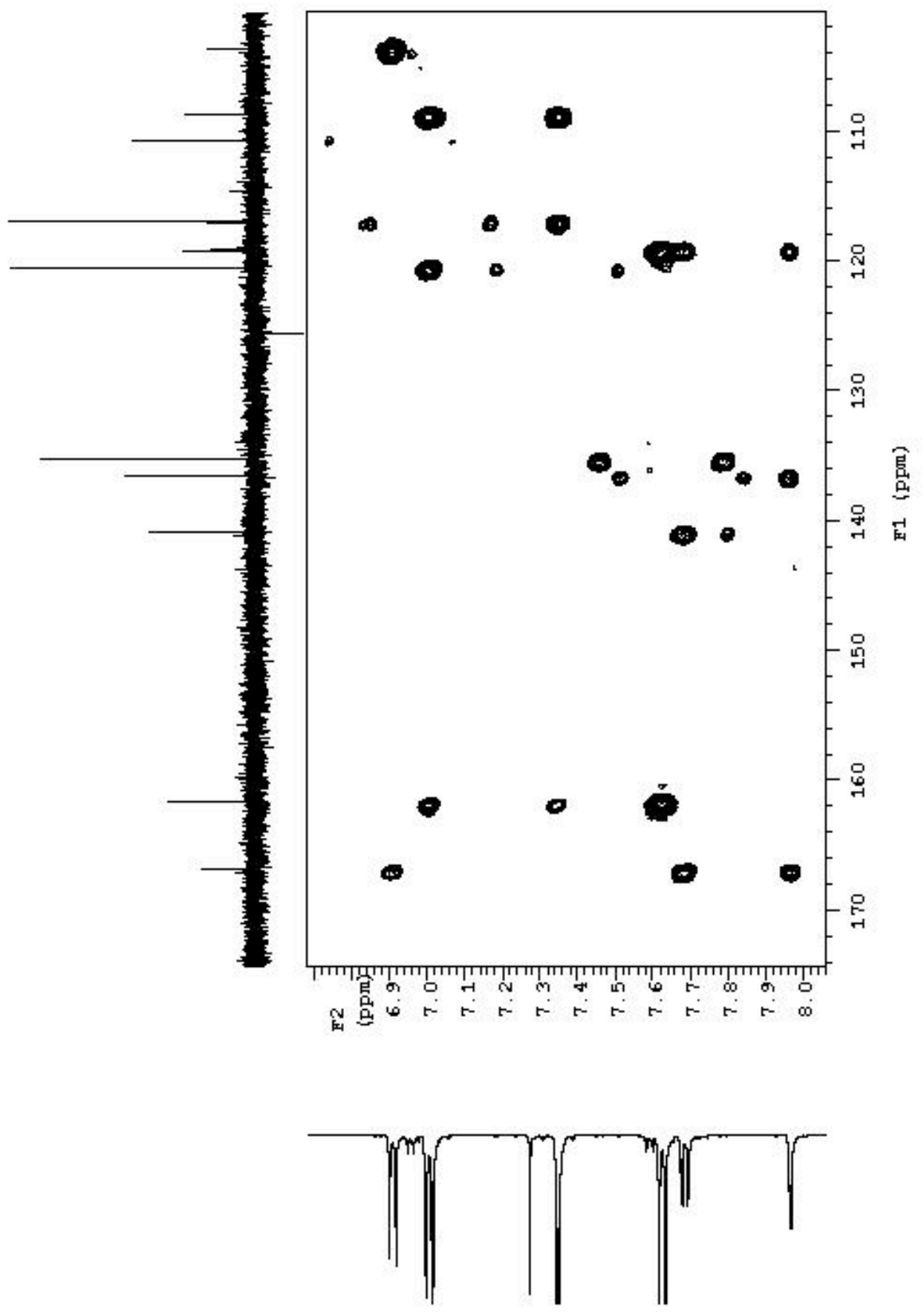

Figure 4. gHMBC spectrum of the borylation products of 4-methoxybenzonitrile. 


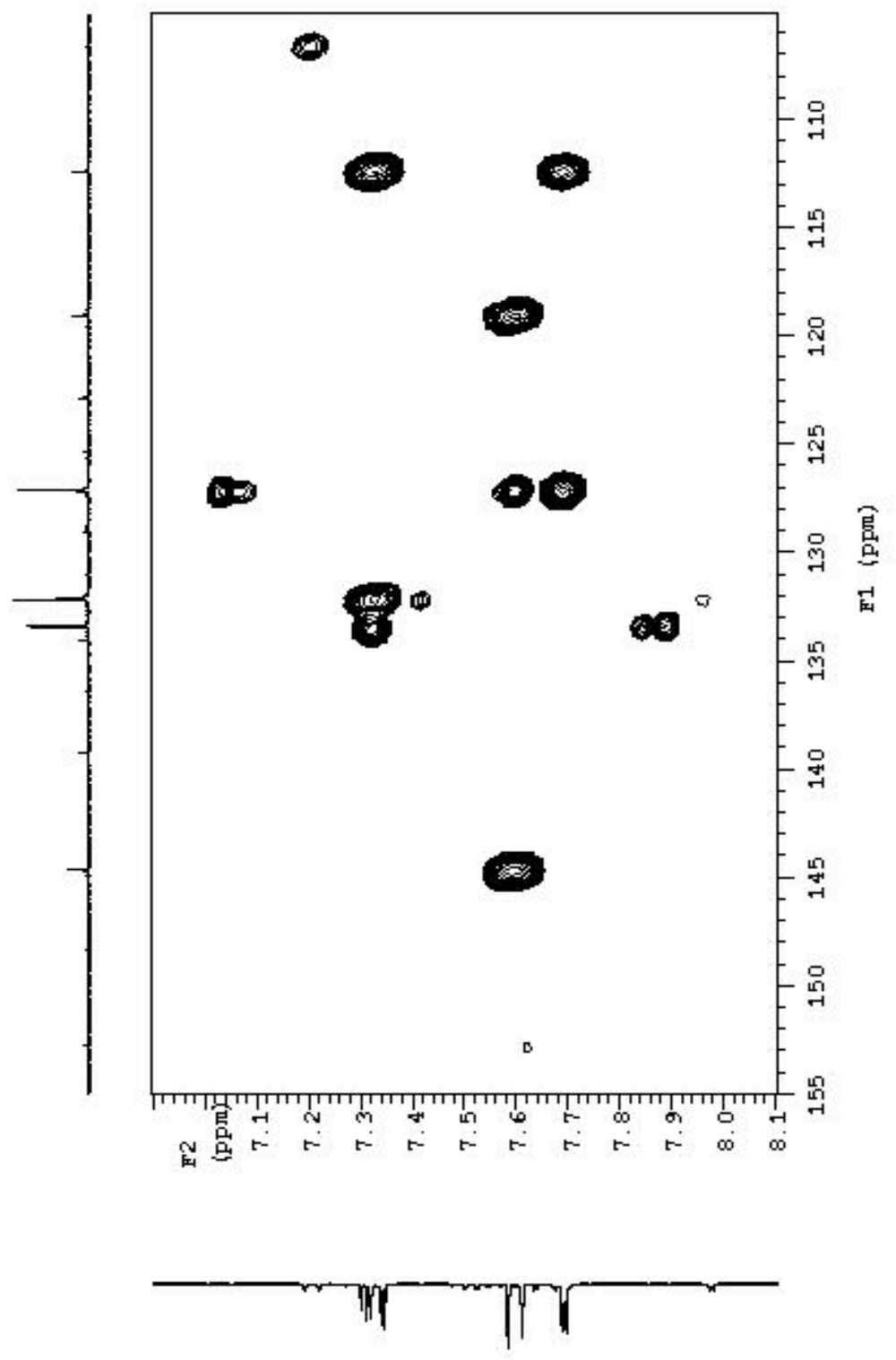

Figure 5. gHMBC spectrum of the borylation products of 4-thiomethylbenzonitrile. 


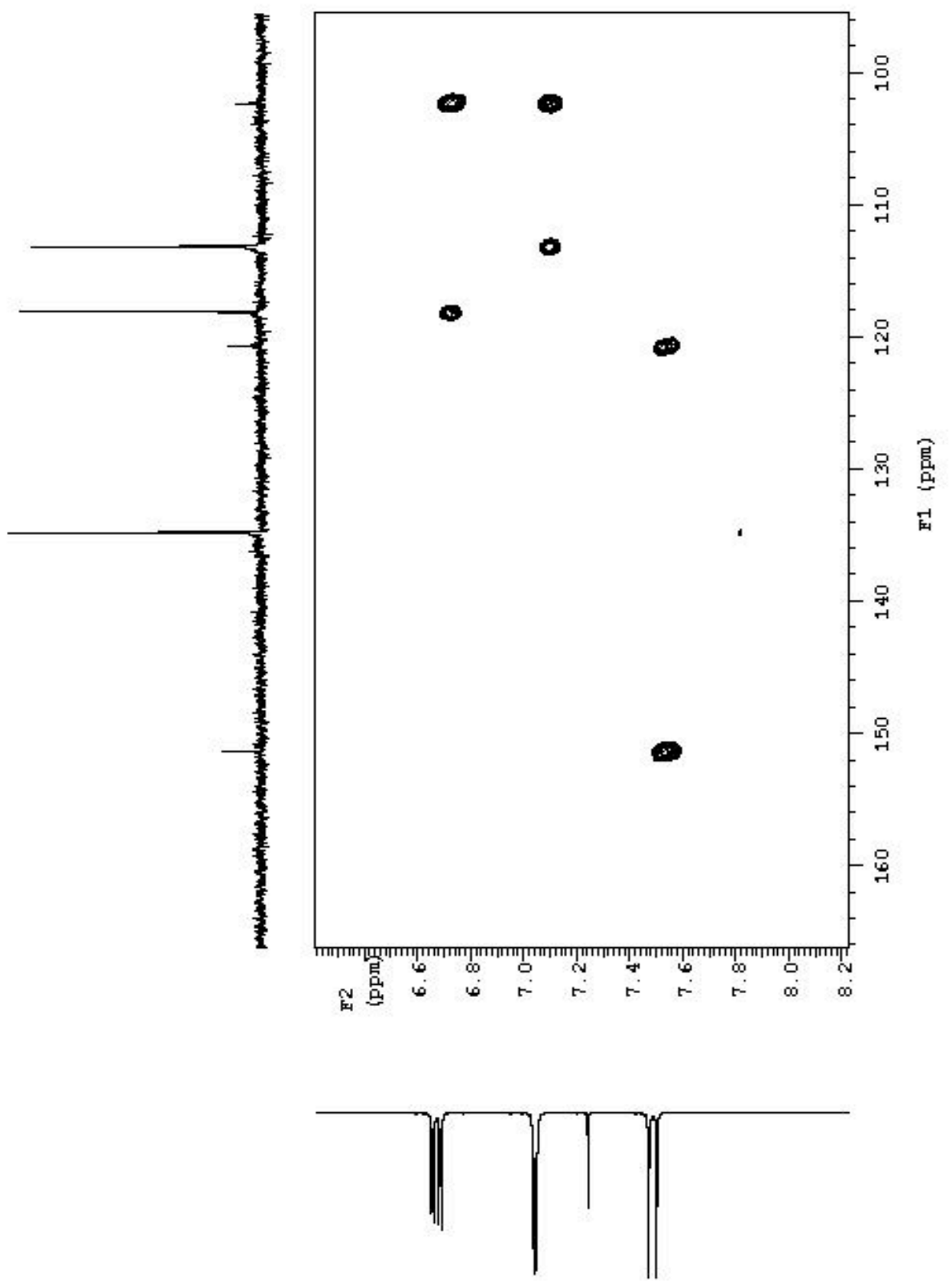

Figure 6. gHMBC spectrum of the borylation product of 4-dimethylaminobenzonitrile. 

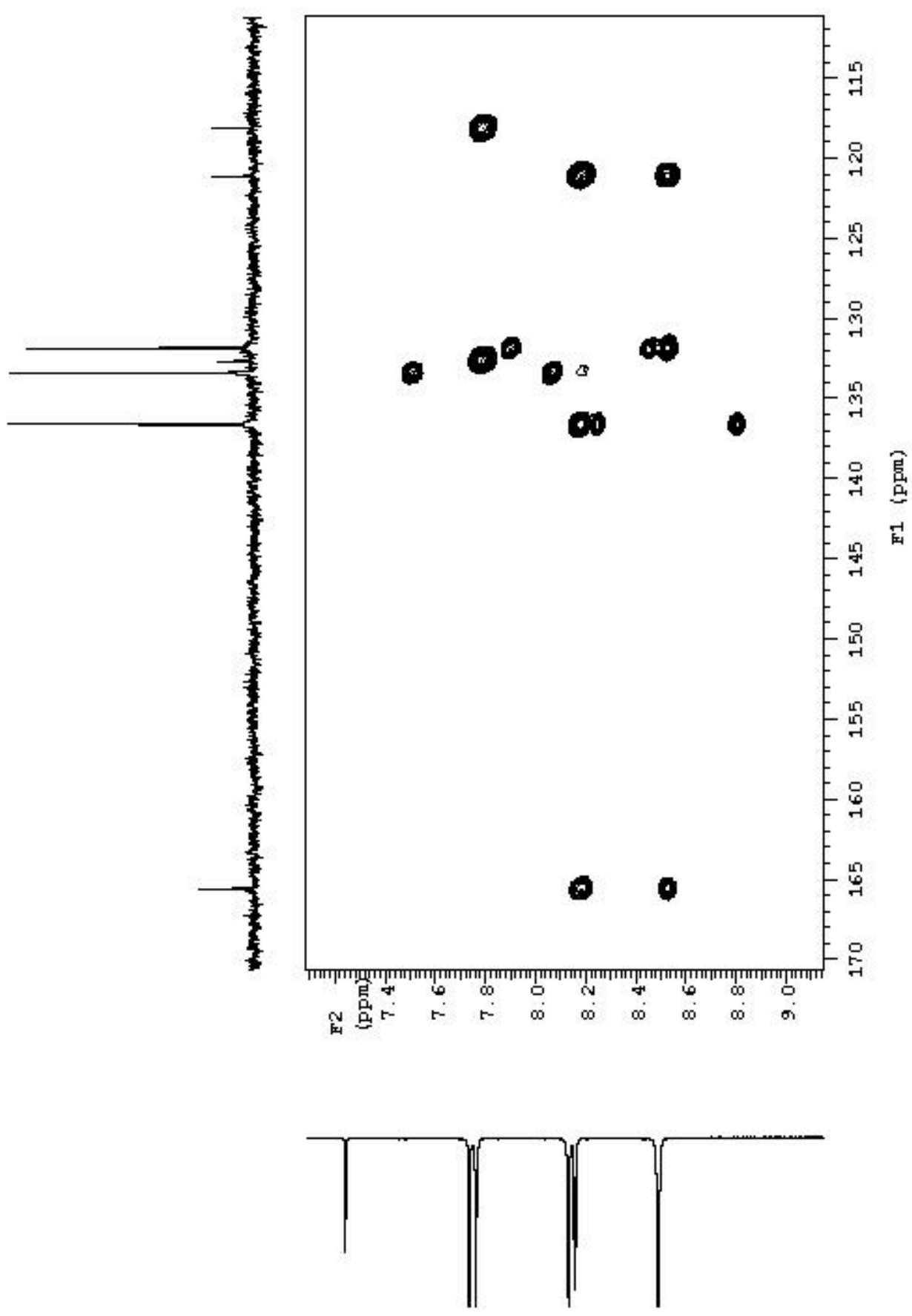

Figure 7. gHMBC spectrum of the borylation product of methyl 4-cyanobenzoate. 


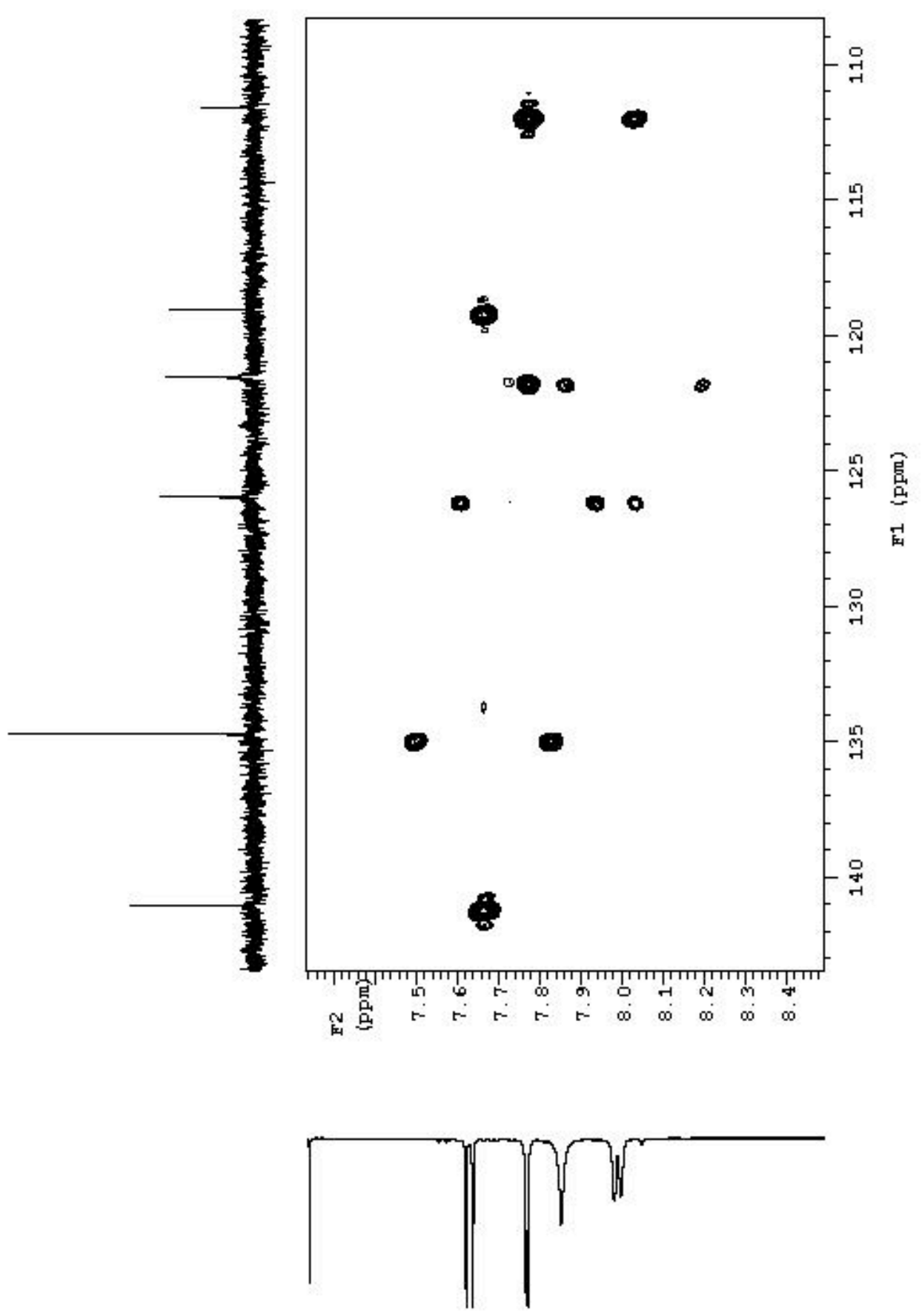

Figure 8. gHMBC spectrum of the borylation product of 4'-cyanoacetanilide. 


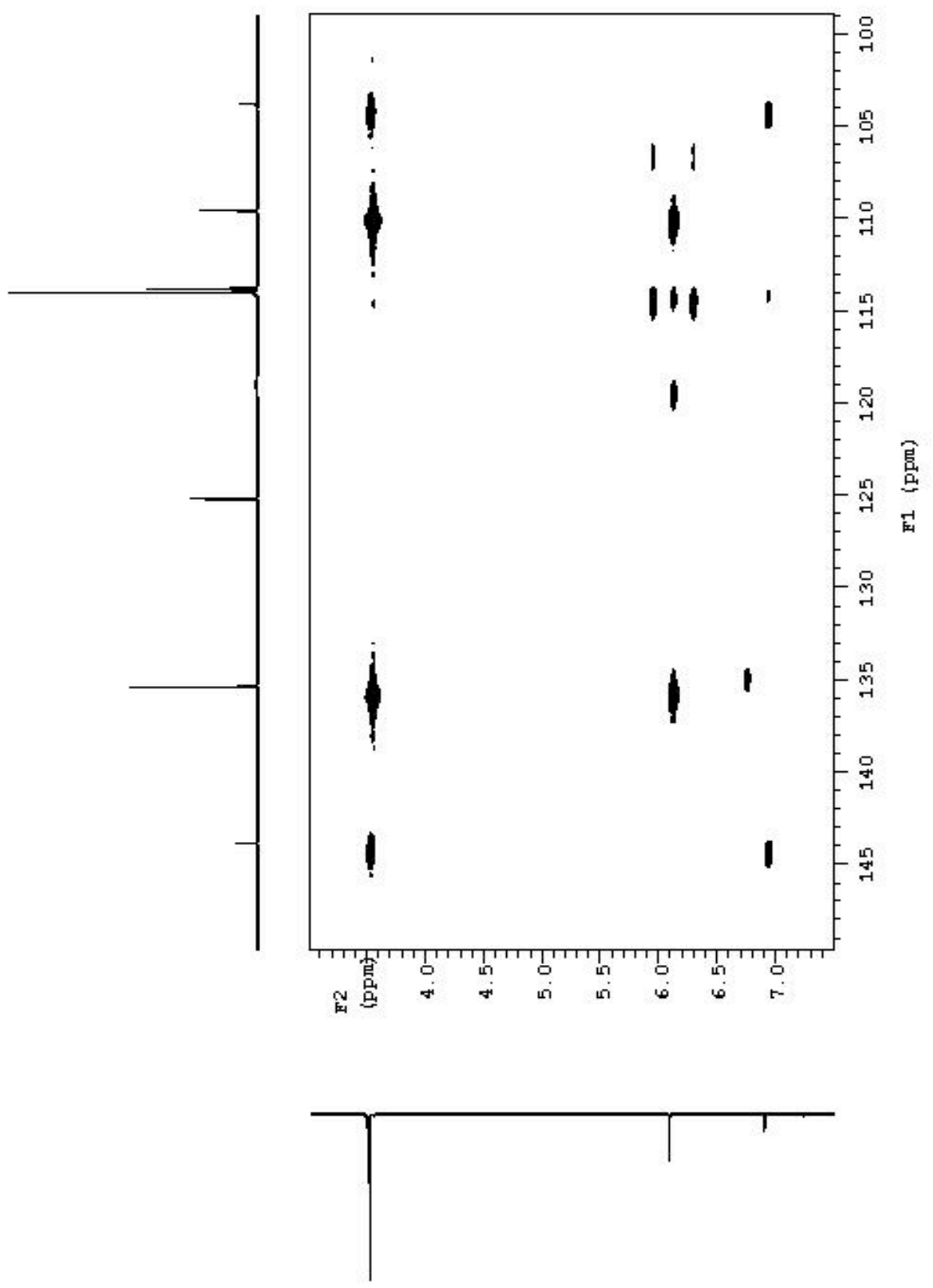

Figure 9. gHMBC spectrum of the borylation products of 1,5-dimethyl-2pyrrolecarbonitrile. 


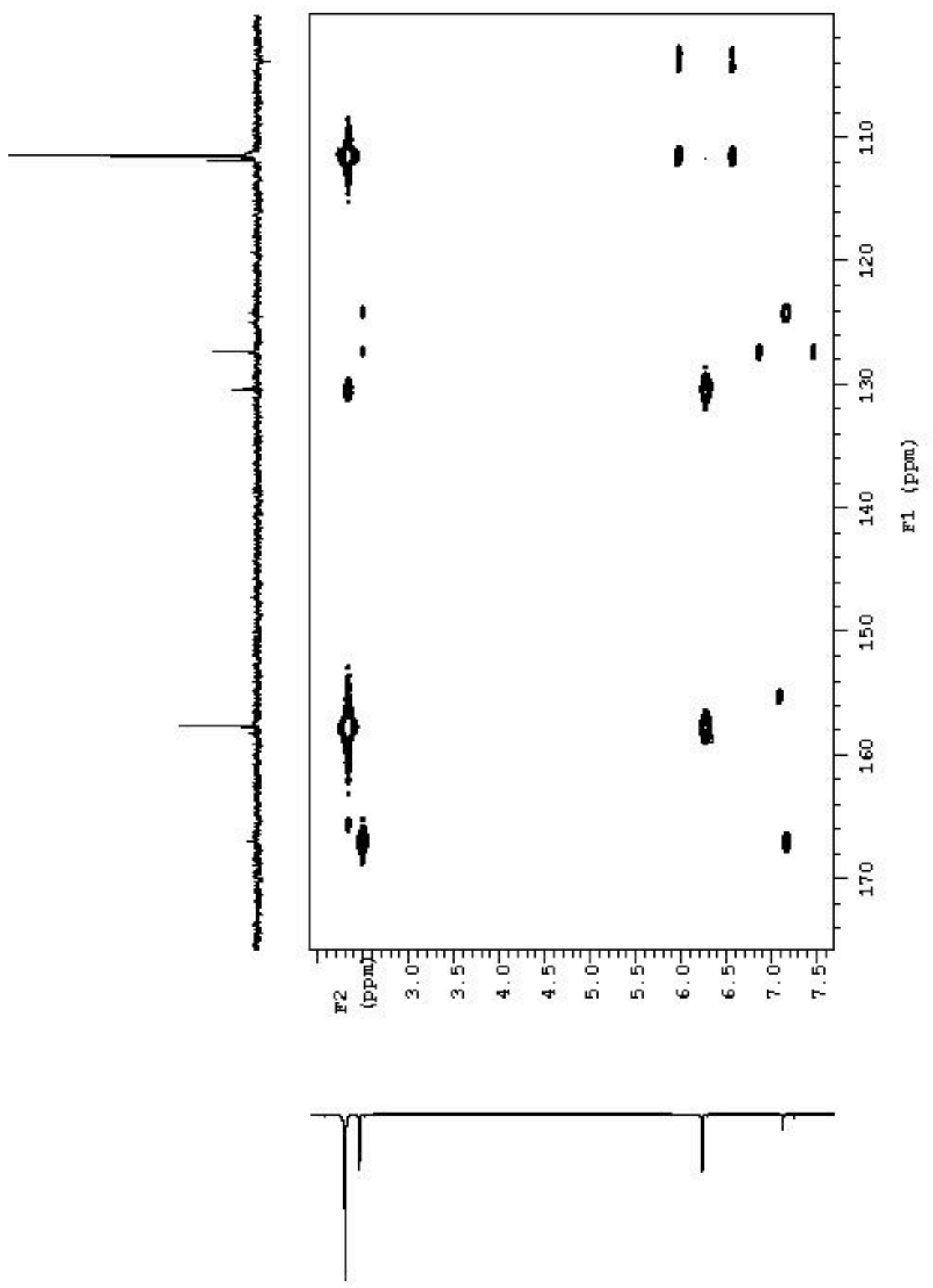

Figure 10. gHMBC spectrum of the borylation products of 5-methyl-2-furonitrile. 